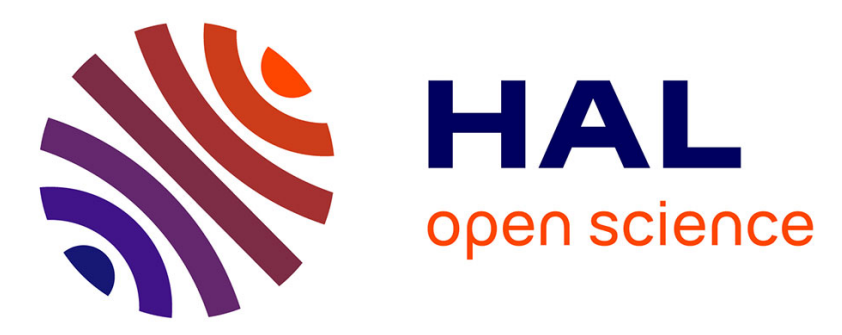

\title{
Near-pure vapor condensation in the Martian atmosphere: CO2 ice crystal growth
}

Constantino Listowski, Anni Määttänen, Ilona Riipinen, Franck Montmessin, Franck Lefèvre

\section{- To cite this version:}

Constantino Listowski, Anni Määttänen, Ilona Riipinen, Franck Montmessin, Franck Lefèvre. Nearpure vapor condensation in the Martian atmosphere: CO2 ice crystal growth. Journal of Geophysical Research. Planets, 2013, 118 (10), pp.2153-2171. 10.1002/jgre.20149 . hal-00861861

\section{HAL Id: hal-00861861 https://hal.science/hal-00861861}

Submitted on 27 Mar 2016

HAL is a multi-disciplinary open access archive for the deposit and dissemination of scientific research documents, whether they are published or not. The documents may come from teaching and research institutions in France or abroad, or from public or private research centers.
L'archive ouverte pluridisciplinaire HAL, est destinée au dépôt et à la diffusion de documents scientifiques de niveau recherche, publiés ou non, émanant des établissements d'enseignement et de recherche français ou étrangers, des laboratoires publics ou privés. 


\title{
Near-pure vapor condensation in the Martian atmosphere: $\mathrm{CO}_{2}$ ice crystal growth
}

\author{
C. Listowski, ${ }^{1}$ A. Määttänen, ${ }^{1}$ I. Riipinen, ${ }^{2}$ F. Montmessin, ${ }^{1}$ and F. Lefèvre ${ }^{3}$ \\ Received 27 November 2012; revised 24 July 2013; accepted 31 August 2013; published 11 October 2013.
}

[1] A new approach is presented to model the condensational growth of carbon dioxide $\left(\mathrm{CO}_{2}\right)$ ice crystals on Mars. These condensates form in very particular conditions. First, $\sim 95 \%$ of the atmosphere is composed of $\mathrm{CO}_{2}$ so that near-pure vapor condensation takes place. Second, the atmosphere is rarefied, having dramatic consequences on the crystal growth. Indeed, the subsequently reduced efficiency of heat transport helps maintain a high temperature difference between the crystal surface and the environment, inhibiting the growth. Besides, the Stefan flow which would have been expected to increase the growth rate of the crystal, because of the near-pure vapor condensation, is negligible. We show that the heritage of the convenient and explicit linearized crystal growth rate formula used for Earth clouds, initially derived for a trace gas, has to be reconsidered in the case of near-pure vapor condensation for high saturation ratios that appear to be common in the Martian mesosphere. Nevertheless, by comparing our approach with a more complex condensation model, valid for all atmospheric conditions and all vapor abundances, we show that a very simple set of equations can still be used to efficiently reproduce the $\mathrm{CO}_{2}$ ice crystal growth rate. Our model, referred to as the CLASSIC model here, provides similar crystal growth rates than the traditionally used linearized growth rate models at low supersaturations but predicts lower crystal growth rates at high supersaturations. It can thus be used to model the condensational growth of $\mathrm{CO}_{2}$ ice crystals in the mesosphere where high supersaturations are observed.

Citation: Listowski, C., A. Määttänen, I. Riipinen, F. Montmessin, and F. Lefèvre (2013), Near-pure vapor condensation in the Martian atmosphere: $\mathrm{CO}_{2}$ ice crystal growth, J. Geophys. Res. Planets, 118, 2153-2171, doi:10.1002/jgre.20149.

\section{Introduction}

[2] Cloud formation on Earth essentially involves water vapor, a trace gas that forms either liquid droplets or ice crystals and sometimes both phases within a so-called mixedphase cloud. Cloud formation on Mars is more simple in that regard as only ice forms during condensation process. While Mars atmosphere does host water-ice clouds as well, formed out of water vapor as a trace gas, another type of clouds made solely of carbon dioxide ice crystals $\left(\mathrm{CO}_{2}\right)$ also exists. Ninety-five percent of the Martian atmosphere consists of $\mathrm{CO}_{2}$ gas, we are thus dealing with the condensation of the main component of an atmosphere, which is a rare phenomenon in the solar system. Note that on Triton, the biggest satellite of Neptune, ice particles formed out of the

\footnotetext{
${ }^{1}$ Université Versailles St-Quentin; UPMC University Paris 06; CNRS/ INSU, LATMOS-IPSL, Guyancourt, France.

${ }^{2}$ Department of Applied Environmental Science and Bert Bolin Center for Climate Research, Stockholm University, Stockholm, Sweden.

${ }^{3}$ UPMC University Paris 06; Université Versailles St-Quentin; CNRS/ INSU, LATMOS-IPSL, Paris, France.

Corresponding author: C. Listowski, Université Versailles St-Quentin; UPMC University Paris 06; CNRS/INSU, LATMOS-IPSL, 11 boulevard dAlembert, 78280 Guyancourt, France. (constantino.listowski@ latmos.ipsl.fr)
}

(C)2013. American Geophysical Union. All Rights Reserved. 2169-9097/13/10.1002/jgre.20149 main atmospheric component (nitrogen $\mathrm{N}_{2}$ ) in a very rarefied atmosphere [Elliot et al., 1998] have been reported as well [see, e.g., Pollack et al., 1990]. Nevertheless, Mars is so far the only telluric planet where formation of clouds out of the main atmospheric component has been observed.

[3] First suggestions of $\mathrm{CO}_{2}$ ice clouds presence in the atmosphere were made by Herr and Pimentel [1970] with the Mariner 6 and 7 spectroscopic detections of a $4.3 \mu \mathrm{m}$ signature. It was most likely due to non-local thermodynamic equilibrium emission of $\mathrm{CO}_{2}$ gas, which is known to occur at that particular wavelength from observations [Lellouch et al., 2000; Drossart et al., 2006] and modeling [López-Valverde et al., 2005]. Moreover, the emission peak was too low $(25 \pm 7 \mathrm{~km})$ for condensation to take place at equatorial latitudes. Clancy and Sandor [1998] combined the former study, their submillimeter observations from Earth and the observations of Mars Pathfinder's (MPF) lander [Smith et al., 1997] to infer that the direct imaging of a bluish cloud in the predawn sky by MPF could actually be a carbon dioxide mesospheric cloud. In addition, Clancy et al. [2003, 2007] produced the first systematic observations of Martian mesospheric clouds using the thermal emission spectrometer onboard the Mars Global Surveyor mission. However, the first unambiguous spectroscopic detection of $\mathrm{CO}_{2}$ ice crystals in the atmosphere was made by Montmessin et al. [2007] with the instrument OMEGA onboard the Mars Express mission. 
[4] $\mathrm{CO}_{2}$ condensation in the Martian atmosphere was also considered in the frame of a warm early Mars where surface temperatures above the freezing point of water could have been sustained due to an efficient greenhouse effect triggered by a denser $\mathrm{CO}_{2}$ atmosphere [Pollack, 1979]. Kasting [1991] argued that if $\mathrm{CO}_{2}$ condensation was taken into account in the atmosphere's energy budget, this ruled out the possibility of an efficient global warming by $\mathrm{CO}_{2}$ gas absorption, in contrary to what previous models were suggesting. However, the author neglected the scattering properties of $\mathrm{CO}_{2}$ ice crystals. Forget and Pierrehumbert [1997] considered in their model the strong scattering efficiency of $\mathrm{CO}_{2}$ ice crystals in the thermal infrared [Warren et al., 1990; Hansen, 1997]. $\mathrm{CO}_{2}$ ice clouds can backscatter the outgoing planetary IR flux toward the surface, and despite the increase in the planetary albedo due to cloud formation, the net effect could have been an increase of the surface temperatures. Mischna et al. [2000] went further into details by investigating the effect of fractional cloud coverage, optical depth, and altitude on the net effect of $\mathrm{CO}_{2}$ ice clouds in an early Martian atmosphere. For instance, a cloud cover greater than $50 \%$ would be needed to maintain temperatures above the freezing point of water. Finally, and as pointed by Mischna et al. [2000] in their conclusion, a detailed microphysics parameterization in a 3-D general circulation model is definitely needed to estimate the actual climatic effect of such clouds. Colaprete and Toon [2003] investigated $\mathrm{CO}_{2}$ cloud formation in an early dense Martian atmosphere with a onedimensional model. They underline the self-limiting effect of cloud formation. The warming of the surface-atmosphere system by the clouds is restrained by the radiative warming and latent heat release which limit their lifetime. Despite an overall increase in surface temperatures, no values above the freezing point of water were obtained. Forget et al. [2013] used a three-dimensional Martian global circulation model (MGCM) including simple microphysics of $\mathrm{CO}_{2}$ ice clouds to study primitive Mars. They found also that no combination of model parameters (obliquity, orbital parameters, cloud microphysics parameters, dust loading, surface properties) could result in temperatures above the freezing point of water except for obliquities larger than $40^{\circ}$ but not in locations with geological evidence for past liquid water.

[5] Today's Martian $\mathrm{CO}_{2}$ ice clouds have been observed by several remote-sensing instruments, yielding constraints on, e.g., particle size that could in turn help models in their climate predictions. $\mathrm{CO}_{2}$ ice clouds are observed in the polar night at tropospheric altitudes $(\lesssim 20 \mathrm{~km})$ [Pettengill and Ford, 2000; Ivanov and Muhleman, 2001; Neumann et al., 2003; Hayne et al., 2012; Hu et al., 2012]. Some mesospheric clouds have been clearly identified as $\mathrm{CO}_{2}$ ice clouds by spectroscopy [Montmessin et al., 2007; Scholten et al., 2010; Määttänen et al., 2010; Vincendon et al., 2011], while for other observations, ambiguities remain about the nature (water or $\mathrm{CO}_{2}$ ice) of these high-altitude crystals [Clancy et al., 2003, 2007; McConnochie et al., 2010].

[6] We recall here that the Martian mesosphere is the transition layer $(\sim 50-100 \mathrm{~km})$ between the troposphere where radiative-convective equilibrium dominates and the thermosphere where temperature increases with height. Thus, it is the atmospheric layer where near-infrared absorption of incoming sunlight by atmospheric $\mathrm{CO}_{2}$ dominates over the convective regime [see, e.g., López-Valverde et al., 2011].
[7] Mesospheric $\mathrm{CO}_{2}$ ice clouds can be divided into two categories. A first set of equatorial clouds is observed during the day, in both hemispheres, in the altitude range $\sim 60-$ $85 \mathrm{~km}$, and with particle sizes around 1-2 $\mu \mathrm{m}$. A second set at subtropical latitudes and higher altitudes ( 90-100 km), during the night, with particle sizes on the order of $0.1 \mu \mathrm{m}$ [Montmessin et al., 2006]. At these mesospheric altitudes, pressure ranges from $\sim 0.1 \mathrm{~Pa}$ to $\sim 0.001 \mathrm{~Pa}$. Regarding polar tropospheric clouds, optical extinctions observed in the polar nights with the Mars Orbiter Laser Altimeter (MOLA) onboard Mars Global Surveyor (MGS) suggest particles around $\lesssim 100 \mu \mathrm{m}$ [Colaprete et al., 2003] at altitudes $\leq 20 \mathrm{~km}$ where atmospheric pressure is several tens of Pa. Recently, Hu et al. [2012] focused on simultaneous retrievals of supersaturated temperature profiles from the Radio Science (RS) experiment onboard MGS and the Mars Climate Sounder (MCS) onboard the Mars Reconnaissance Orbiter (MRO) and detection of reflective clouds by MOLA in the poles. They derived particle size at the top of the clouds in the range of 8-22 $\mu \mathrm{m}$ for the $\mathrm{CO}_{2}$ condensates in the northern hemisphere and 4-13 $\mu \mathrm{m}$ in the southern hemisphere. Hayne et al.'s [2012] estimations in terms of particle size only allow to constrain the range of effective radii $(1-100 \mu \mathrm{m})$ that might be expected along the vertical in the south pole (below $30 \mathrm{~km})$.

[8] Polar $\mathrm{CO}_{2}$ ice clouds have been well reproduced by models as mountain wave clouds [Tobie et al., 2003] or alternatively as clouds forming through a "moist convection" process [Colaprete et al., 2008], where the large latent heat release due to $\mathrm{CO}_{2}$ ice crystal formation fuels strong convective vertical motions. Colaprete et al. [2008] describe these clouds as huge convective formations for which only a very few observational constraints exist. Colaprete et al. [2008] modeled particle radii around $30-80 \mu \mathrm{m}$ in the polar regions and Tobie et al. [2003] modeled wave clouds with maximum average crystal radii in the range $60-180 \mu \mathrm{m}$ depending on their assumptions on winds and number nuclei. These modeled particle sizes are bigger than the $\sim 22 \mu \mathrm{m}$ particles observed by $\mathrm{Hu}$ et al. [2012]. It is important to note that $H u$ et al. [2012] derived particle sizes at the top of the clouds and bigger ice crystals are to be expected at lower altitudes. Regarding the mesospheric clouds, Colaprete et al.'s [2008] study is the first and only attempt so far to investigate these clouds by coupling a $\mathrm{CO}_{2}$ ice cloud microphysical scheme to a MGCM. Their simulations match the typical opacities ( $\sim 0.02-0.1)$ observed for these clouds, and they modeled crystal radii in the range $\leq 1-10 \mu \mathrm{m}$ at $60-70 \mathrm{~km}$ which is close although bigger than the typical radii $(\sim 1-2 \mu \mathrm{m})$ observed up to $\sim 85 \mathrm{~km}$ into the mesosphere [Montmessin et al., 2007; Määttänen et al., 2010; Vincendon et al., 2011]. Regarding the altitudes, most of the simulated mesospheric clouds in the $60^{\circ} \mathrm{S}, 60^{\circ} \mathrm{N}$ region lie between the $0.03 \mathrm{mbar}$ $(\sim 60 \mathrm{~km})$ and $0.01 \mathrm{mbar}(\sim 70 \mathrm{~km})$ levels where $\mathrm{CO}_{2}$ ice clouds have been observed. However, there are discrepancies between the model and the observations regarding the spatial and seasonal distributions. Smaller crystal radii, as derived for the highest detected clouds by Montmessin et al. [2006] $(0.1 \mu \mathrm{m}$ at $\sim 100 \mathrm{~km})$, could not be obtained at the altitudes explored by the model (lower than $85 \mathrm{~km}$ ). However, as recalled by Colaprete et al. [2008], the crystal sizes resulting from condensational growth are also a matter of the assumed number of available nuclei (e.g., meteoritic dust 
or mineral dust), which can serve as substrate for heterogeneous nucleation [Määttänen et al., 2005]. $\mathrm{CO}_{2}$ ice cloud modeling that involves the condensation of the major atmospheric component has turned out to be challenging and the nature of the key microphysical processes in the formation of $\mathrm{CO}_{2}$ ice clouds remains unclear, especially in the mesosphere.

[9] Recent modeling studies suggest that the effect of the thermal tide in cooling the mesosphere is a prerequisite for $\mathrm{CO}_{2}$ cloud formation [González-Galindo et al., 2011], with smaller-scale perturbations required to reach the $\mathrm{CO}_{2}$ condensation temperatures. A recent study has shown a strong correlation between mesospheric ice cloud observations and the propagation of gravity waves through the atmosphere [Spiga et al., 2012]. These waves could create cold pockets in which $T \leq T_{\text {cond }}$, and thus create a supersaturated environment in which the clouds can form. Most of the mesospheric clouds that have been spectroscopically identified as $\mathrm{CO}_{2}$ ice clouds are located in the equatorial region $\left(30^{\circ} \mathrm{S}, 30^{\circ} \mathrm{N}\right)$ [Scholten et al., 2010; Määttänen et al., 2010; Vincendon et al., 2011]. In these regions at mesospheric altitudes, temperature excursions below the condensation point of $\mathrm{CO}_{2}$ have been measured by [Schofield et al., 1997] with the Mars Pathfinder entry descent and landing experiment at $\sim 80 \mathrm{~km}$ altitude, by Montmessin et al. [2006] at $\sim 100 \mathrm{~km}$, Forget et al. [2009] around the mesopause $(p \sim 0.01-0.0001 \mathrm{~Pa})$, and Montmessin et al. [2011], all three of them with the SPICAM instrument on board Mars Express. These cold pockets appear to be common in the Martian mesosphere. In the highest layers of the mesosphere temperatures as low as $24 \mathrm{~K}$ below the condensation temperature of $\mathrm{CO}_{2}$ were reported. For a $95 \% \mathrm{CO}_{2}$ abundance, at pressures $p<0.01 \mathrm{~Pa}$, this is equivalent to saturation ratios $S>1000$. In this paper we focus on theoretical aspects of the $\mathrm{CO}_{2}$ ice crystal growth in that extreme Martian environment. Microphysics is indeed a first step to understand the $\mathrm{CO}_{2}$ ice clouds on Mars, although coupling a microphysical model to a mesoscale circulation model would be further needed to explain the mesospheric cloud observations.

[10] First introducing the two main ways to describe the growth of an ice crystal, we rely on the two most recent studies on modeling the $\mathrm{CO}_{2}$ ice crystal growth, Colaprete and Toon [2003] for early Mars' clouds and Wood [1999] for polar $\mathrm{CO}_{2}$ snow in current Mars. The former model was used by Colaprete et al. [2003] and Colaprete et al. [2008], respectively, for southern polar $\mathrm{CO}_{2}$ ice clouds and polar and mesospheric $\mathrm{CO}_{2}$ ice clouds in the current Martian atmosphere. We explain how the two standard models account in different ways for the influence of the rarefied atmosphere on the growth rate. In addition to the physics used by Colaprete and Toon [2003], we investigate processes like Stefan flow (enhancement term in the mass flux), thermal mass diffusion (influence of the temperature gradient on the mass flux), and Dufour effect (influence of the partial pressure gradient on the heat flux). We then present our reference model. We show that usual trace gas growth models inherited from Earth cloud microphysics cannot be applied to near-pure vapor condensation in a highly supersaturated environment as the Martian mesosphere can be. We compare our growth rates to the condensation scheme developed by Young [1993] and used by Wood [1999]. We show that whatever the saturation ratios, we obtain the same growth rates as the ones derived from Young's [1993] model which includes more physical processes than our reference model, and we explain that match.

\section{Theory: From a Trace Gas to a Near-Pure Vapor}

[11] We assume spherical symmetry for the crystal and thus apply the same equations as we would for a droplet. We only focus on the condensation process but nucleation is the prerequisite to any subsequent growth of the newly formed droplet or crystal. Ice nucleation consists in the formation of a so-called critical ice embryo from the vapor phase or the liquid phase. On Earth, it is most often the latter. On Mars, ice nucleation occurs from the vapor phase (in lack of any stable liquid phase), and it is most likely heterogeneous [Määttänen et al., 2005] in opposition to homogeneous nucleation which consists in formation of the new phase in the mother phase without any substrate. These substrates may be provided by dust particles, ions, or previously formed water ice crystals. Dust particles may be mineral dust lifted from the ground by winds as well as micrometeorites. As nucleation is not the topic of this paper, we consider that the critical ice embryo has formed. Note that we will neither focus on surface kinetics effects nor on radiative heat transfer. We exclusively focus on the condensation process as it was considered by Colaprete and Toon [2003]; Colaprete et al. [2003, 2008] (with the radiative heat transfer term set to zero and no surface kinetics effect). Also, Wood [1999] did not consider the radiative heat transfer for the different growth rates he presents in his work (see our discussion in section 3.3.1).

\subsection{Conceptual Models for Crystal Growth}

[12] Two regimes are mentioned when dealing with the growth (or evaporation) of a crystal (or a droplet). They are defined as "free molecular regime" (kinetic regime) and "continuum regime." It is the value of the mean free path $\lambda$ of the diffusing molecules compared to the crystal radius $a$ that determines in which of the two regimes the condensation (evaporation) takes place. One uses the dimensionless Knudsen number $(K n=\lambda / a)$ to determine which description is relevant. If $K n \gg 1$ then the regime is kinetic and one can use the simple Hertz-Knudsen equation [see, e.g., Pruppacher and Klett, 2010] to derive the mass flux toward the particle, which requires the mean thermal velocity of a molecule. If $K n \ll 1$, the regime is a continuum and the laws of diffusion apply. At $0.01<K n<100$, a transition regime takes place. In a transition regime, neither a pure continuum nor a pure kinetic description is applicable. On Mars, given the low densities $(\lambda \gtrsim 10 \mu \mathrm{m})$ and small crystal sizes $(a \lesssim 100 \mu \mathrm{m})$, the crystals growing in the atmosphere lie either in the transition or in the kinetic regimes ( $K n$ always $\gtrsim 0.1$ ).

[13] Two descriptions of the growing crystal can be used: a "Maxwell type" or a "Langmuir type." Figure 1 summarizes these two approaches. A Maxwell-type model will assume that pure continuum prevails around the crystal so that Fick's law for diffusion and Fourier's law for heat conduction apply everywhere. Maxwell [1890] used this model first to model the growth of water droplets. The partial pressure of the condensing vapor at the crystal surface is 


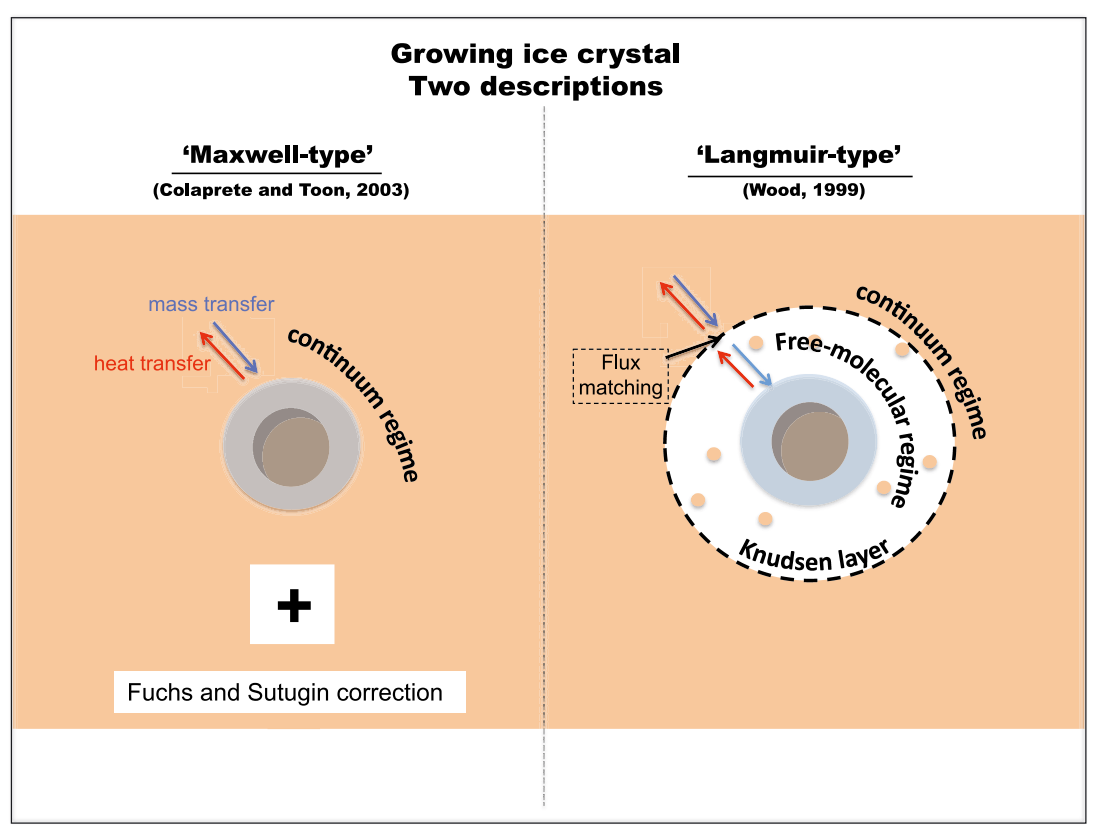

Figure 1. An illustration of the two approaches that can be used to model the crystal growth in environments where no strict continuum prevails (i.e., the Knudsen number $K n$ is no longer $\ll 1$ ). The figure shows (left) a Maxwell-type description where continuum is assumed all around the crystal. An effective correction of the transport properties (namely, the thermal conductivity $K$ and the diffusion coefficient $D$ ) is thus mandatory. This correction is not needed in (right) a Langmuir-type approach where one considers a Knudsen layer around the crystal where free molecular regime is assumed. This Knudsen layer is in contact with the background medium, assumed as a continuum and where fluid mechanics applies. Two recent models of $\mathrm{CO}_{2}$ ice crystal growth used one of two approaches: Colaprete and Toon [2003] use the Fuchs and Sutugin correction in a Maxwell-type approach, relying on Toon et al.'s [1989] work, while Wood [1999] uses the model of Young [1993] which relies on a Langmuir-type approach with a more complete set of equations than Toon et al. [1989]. Both models are discussed in this work.

assumed to be the saturation vapor pressure $p_{\text {sat }}$. Colaprete and Toon [2003, hereafter C2003] used this approach for $\mathrm{CO}_{2}$ ice crystal growth with an effective correction on transport properties to account for the noncontinuum effects (see further). A Langmuir-type model distinguishes a so-called Knudsen layer of thickness $\sim \lambda$, between the crystal surface and the surroundings, where kinetic theory of gases prevails [Langmuir, 1915]. Beyond that layer, far from the condensate, a continuum region is assumed and fluid mechanics can be applied. This approach accounts for the possibility of a transition to kinetic regime, the Knudsen layer depth depending on the value of $\lambda$, i.e., on the atmospheric density. Wood [1999, hereafter W1999] used a condensational growth model relying on this description of the system [Young, 1991, 1993]. No regime-dependent corrections are needed as all regimes are taken into account within this approach. Conversely, the Maxwell-type model is not in itself suitable to transition or kinetic regime and needs some corrections. This correction concerns the transport coefficient, namely the diffusion coefficient $D$ and the thermal conductivity $K$ defined for continuum regimes. Both are reduced at higher Knudsen numbers (low densities and small particles) where transport is less efficient. Different formulas exist for this correction [see, e.g., Seinfeld and Pandis, 2006, Table 12.1]. One of these is the correction proposed by Fuchs and Sutugin [1971] that is used in a broad range of studies, such as Toon et al. [1989] for polar stratospheric clouds, Colaprete and Toon [2003] for $\mathrm{CO}_{2}$ ice clouds on Mars, or Montmessin et al. [2002] for water ice clouds on Mars. We comment in section 3.3.4 on the choice of this specific correction. The correction is defined as follows:

$$
D^{\prime}=\frac{D}{1+f(K n)} \text { and } K^{\prime}=\frac{K}{1+f(K n)}
$$

where $D$ and $K$ are, respectively, the diffusion coefficient and the thermal conductivity in continuum regime and $D^{\prime}$ and $K^{\prime}$ the corresponding corrected coefficients, with $f(K n)$ being defined as

$$
f(K n)=K n \frac{1.333+0.71 K n^{-1}}{1+K n^{-1}}
$$

[14] This function $f$ is such that $f(K n \rightarrow 0)=0$ and $f(K n \rightarrow \infty)=\infty$.

[15] The Knudsen number has two different definitions whether it is used for diffusion $\left(K_{n, d}\right)$ or heat conduction $\left(K_{n, h}\right)$. We use the same definitions as Toon et al. [1989]:

$$
\begin{gathered}
K_{n, d}=\frac{\lambda_{d}}{a}=\frac{3 D}{v_{C O_{2}}^{t h} a}, \\
K_{n, h}=\frac{\lambda_{h}}{a}=\frac{3 K}{\rho_{\text {air }} v_{\text {air }}^{t h}\left(C_{p, \text { air }}-0.5 R_{\text {air }}\right) a},
\end{gathered}
$$

where $a$ is the crystal radius, $v_{\mathrm{CO}_{2}}^{\text {th }}$ is the mean thermal velocity $\left(\mathrm{m} \mathrm{s}^{-1}\right)$ of a $\mathrm{CO}_{2}$ molecule, $\rho_{\text {air }}$ is the air density $\left(\mathrm{kg} \mathrm{m}^{-3}\right)$, 
$\mathrm{C}_{p \text {,air }}$ the specific heat capacity of the air $\left(\mathrm{J} \mathrm{kg}^{-1} \mathrm{~K}^{-1}\right)$ taken as the weighted average of pure $\mathrm{CO}_{2}$ specific heat capacity $\left(\sim 0.7 \times 10^{3} \mathrm{~J} \mathrm{~kg}^{-1} \mathrm{~K}^{-1}\right)$ and $\mathrm{N}_{2}$ specific heat capacity $\left(\sim 1.0 \times 10^{3} \mathrm{~J} \mathrm{~kg}^{-1} \mathrm{~K}^{-1}\right), R_{\text {air }}$ the specific gas constant of the air equal to $R / M_{\text {air }}$ where $M_{\text {air }}$ is the weighted average molar mass of the air mixture, which for Mars is defined in this study as a combination of $\mathrm{CO}_{2}$ and $\mathrm{N}_{2}$ (see section 2.2.1). In this work $\mathrm{CO}_{2}$ is considered as an ideal gas, and so will be its companion vapor $\mathrm{N}_{2}$. Deviations from ideal gas law would have to be considered only at pressures of the order of MPas and higher [Kasting, 1991].

\subsection{Additional Processes for a Near-Pure Vapor in a Maxwell-Type Approach}

[16] We are interested in deriving the mass transfer rate of the condensing vapor $I_{m}\left(\mathrm{~kg} \mathrm{~s}^{-1}\right)$, which is directly related to the crystal growth rate $\mathrm{d} r / \mathrm{d} t$ as follows:

$$
\frac{\mathrm{d} r}{\mathrm{~d} t}=-\frac{1}{4 \pi r^{2} \rho_{\mathrm{ice}}} I_{m},
$$

where $\rho_{\text {ice }}$ is the density of $\mathrm{CO}_{2}$ ice $\left(\mathrm{kg} \mathrm{m}^{-3}\right)$ and $r$ the crystal radius. The minus sign on the right-hand side of the equation is added to have $\mathrm{d} r / \mathrm{d} t>0$ when the crystal grows (corresponding to $I_{m}<0$ ). In the frame of a Maxwell-type approach (like C2003), we investigate processes that are usually neglected for trace gases but that could be of importance on Mars for $\mathrm{CO}_{2}$ ice crystal formation. From now on, we consider implicit the inclusion of the Fuchs and Sutugin correction in the diffusion coefficient $D$ and the thermal conductivity $K$.

\subsubsection{The Basic Equations Used for a Trace Gas}

[17] Following W1999 and C2003, we consider a 95\% $\mathrm{CO}_{2}$ and $5 \% \mathrm{~N}_{2}$ atmosphere with $\mathrm{CO}_{2}$ as the condensible vapor and $\mathrm{N}_{2}$ as the inert gas $\left(\mathrm{N}_{2}\right.$ does not condense at Martian temperatures). The inert $5 \%$ of the Martian atmosphere actually contains $\sim 2.7 \% \mathrm{~N}_{2}$ and $\sim 1.6 \% \mathrm{Ar}$ and other trace gases [Owen, 1992] but to simplify matters and enable a direct comparison, we assume $5 \% \mathrm{~N}_{2}$ (a ternary mixture $\mathrm{CO}_{2} / \mathrm{N}_{2} /$ Ar would mainly affect the thermal conductivity but only to a very small extent, see section 2.3.2.2). Note that in a pure vapor, there is no concentration gradient induced diffusion, and in the Martian case, with $95 \% \mathrm{CO}_{2}$, we are very near the pure vapor limit. However, for inert gas abundances between 5 and $25 \%$, as suggested by remote-sensing observation on Mars [Sprague et al., 2007], it is perfectly correct to apply diffusion laws to $\mathrm{CO}_{2}$ condensation.

[18] In the case of a trace gas $\left(x_{v} \ll 1\right.$, where $x_{v}$ is the mole fraction of the condensing vapor), the related mass transfer rate $I_{m}\left(\mathrm{~kg} \mathrm{~s}^{-1}\right)$ away from the crystal, assumed as having a spherical shape and a radius $a$, results from the Fick's law integration and is given by

$$
I_{m}=-\frac{4 \pi a p M_{v} D}{R T_{\infty}}\left(x_{v, \infty}-x_{v, a}\right),
$$

where $M_{v}$ is the molar mass of the vapor, $R$ the gas constant, $p$ the total pressure, $T_{\infty}$ the background temperature, and $D$ the diffusion coefficient $\left(\mathrm{m}^{2} \mathrm{~s}^{-1}\right) ; x_{v, \infty}$ and $x_{v, a}$ are the mole fractions of condensible vapor far from the crystal and at the crystal surface, respectively. In the following, we refer to $x_{v, \infty}$ as the background mole fraction and to $x_{v, a}$ as the surface mole fraction. Corresponding background and surface partial pressures of the vapor take respectively the form $p_{v, \infty}=x_{v, \infty} p$ and $p_{v, a}=x_{v, a} p$. The mole fraction difference $\left(x_{v, \infty}-x_{v, a}\right)$ drives the mass flux and is positive when condensational growth is occurring $\left(I_{m}<0\right)$. The heat flux $\left(\mathrm{J} \mathrm{s}^{-1}\right)$ results from the Fourier's law and is given by

$$
I_{h}=-4 \pi a^{2} K\left(\frac{\mathrm{d} T}{\mathrm{~d} r}\right)_{r=a}=4 \pi a K\left(T_{a}-T_{\infty}\right),
$$

where $K$ is the thermal conductivity $\left(\mathrm{W} \mathrm{m}^{-1} \mathrm{~K}^{-1}\right.$ ) and $T_{a}$ the temperature at the crystal surface, referred to as the surface temperature in the following. In the case of condensation, heat is released at the crystal surface and is then carried away from the crystal by thermal conduction $\left(I_{h}\right.$ is $\left.>0\right)$. By convention, fluxes directed toward the crystal are considered negative.

\subsubsection{The Stefan Flow}

[19] For the $\mathrm{CO}_{2}$ (vapor)/ $\mathrm{N}_{2}$ (gas) binary system, the mass flux density by diffusion takes the general form [Bird et al., 1960]:

$$
\overrightarrow{j_{v}}=x_{v}\left(\overrightarrow{j_{v}}+\overrightarrow{j_{g}}\right)-\rho D \nabla x_{v},
$$

where $\overrightarrow{j_{v}}$ and $\overrightarrow{j_{g}}$ are the mass flux densities of condensible $\left(\mathrm{CO}_{2}\right)$ and inert gas $\left(\mathrm{N}_{2}\right)$, respectively $\left(\mathrm{kg} \mathrm{m}^{-2} \mathrm{~s}^{-1}\right), x_{v}$ is the mole fraction of the vapor, and $\rho$ is the total mass concentration of the mixture $\left(\mathrm{kg} \mathrm{m}^{-3}\right)$. The first term on the right-hand side can be seen as a convective or macroscopic flow, in addition to the usual gradient-induced flow (the second term on the right). The gradient-induced flow is always related to the average movement of the fluid mixture. An abundant condensible vapor will enhance that average movement and thus the total flux of vapor $\overrightarrow{j_{v}}$. This is why an entrainment term, known as the Stefan flow, has to be considered in addition to the gradient-induced flow, in the case of a nearpure vapor condensing. Because $\mathrm{N}_{2}$ is an inert gas, $\overrightarrow{j_{g}}=0$, resulting in the following simplified mass flux density of vapor:

$$
\overrightarrow{j_{v}}=-\frac{\rho D \nabla x_{v}}{1-x_{v}} .
$$

[20] In the case of a trace gas $\left(x_{v} \ll 1\right)$, equation (7) reduces to the simplified expression (Fick's law of diffusion) used in Earth crystal growth model for condensation of $\mathrm{H}_{2} \mathrm{O}$ : $\overrightarrow{j_{v}}=-c D \nabla x_{v}$ that leads to equation (4) for the mass transfer rate $I_{m}$ after integration over a spherical surface.

[21] The integrated condensing mass transfer rate $I_{m, S F}$ (in $\mathrm{kg} \mathrm{s}^{-1}$ ) which includes the Stefan flow comes from equation (7) and takes the form:

$$
I_{m, S F}=-\frac{4 \pi a p M_{v} D}{R T_{\infty}} \ln \left(\frac{1-x_{v, a}}{1-x_{v, \infty}}\right) .
$$

[22] In Figure 2 the ratio of the mass transfer rate with and without the Stefan flow $\left(I_{m, S F} / I_{m}\right)$ is plotted as a function of the background mole fraction of vapor $x_{v, \infty}$, for different surface vapor mole fraction $x_{v, a}$. For a mole fraction $x_{v, \infty} \sim$ $95 \%$, which is the case for $\mathrm{CO}_{2}$ on Mars, the Stefan flow increases the mass transfer rate up to 40 times the value derived from the trace gas case. However, here we do not account for the heat transfer. This justifies the need of testing the importance of Stefan flow, when coupling the mass transfer with the heat transfer (section 2.3). 


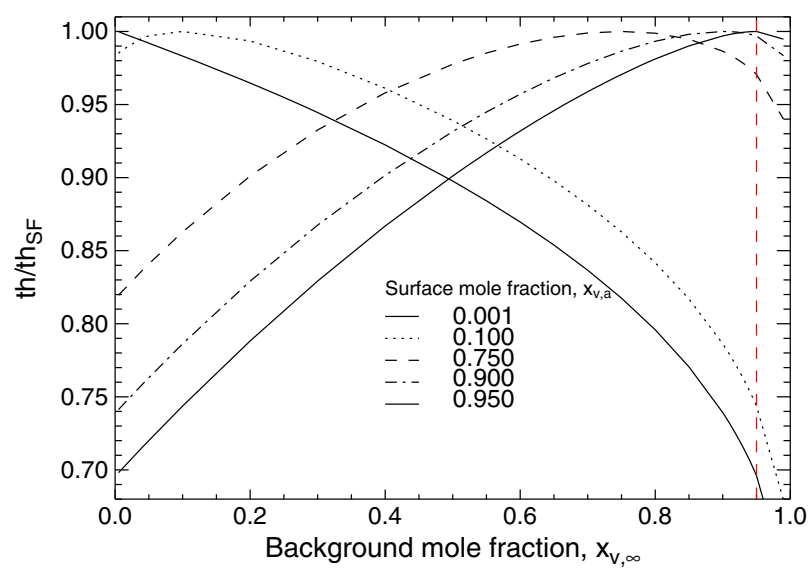

Figure 2. Ratios of the mass transfer rate $\left(\mathrm{kg} \mathrm{s}^{-1}\right)$ accounting for the Stefan flow $I_{m, S F}$ (equation (8)) and the mass transfer rate for a trace gas $I_{m}$ (equation (4)). Ratios are plotted as a function of the background mole fraction of condensible vapor $x_{v, \infty}$ for different mole fractions of vapor at the crystal surface $x_{v, a}: 1 \%$ (solid line), 50\% (dashed line), 90\% (dot-dashed line), and 95\% (triple-dot-dashed line). The vertical dashed line matches the average $95 \% \mathrm{CO}_{2}$ present in the Martian atmosphere.

\subsubsection{Thermal Mass Diffusion and Dufour Effect}

[23] We investigate here the possible influence of thermal mass diffusion and Dufour effect on the mass transfer rate following the work of Kulmala and Vesala [1991] who were interested in water droplet growth in Earth tropospheric conditions. Thermal mass diffusion and Dufour effect rely on the same thermal mass diffusion factor so that both processes have to be considered at the same time.

\subsubsection{Thermal Mass Diffusion}

[24] Thermal mass diffusion is the effect of the temperature gradient on the mass flux density. Even when there are no concentration gradients (e.g., in the case where $x_{v}=$ $100 \%$ ), the temperature gradient can cause diffusion of species. This process is also know as the "Soret effect" in the case of liquid mixtures. The mass flux density defined in equation (6), to which the thermal mass diffusion is added, becomes

$$
\overrightarrow{j_{v}}=-\frac{D M_{v} p}{R T\left(1-x_{v}\right)}\left(\nabla x_{v}+\frac{\alpha x_{v}\left(1-x_{v}\right)}{T} \nabla T\right),
$$

where $\alpha$ is the thermal mass diffusion factor of the vapor, equal to $k_{T} /\left(x_{v} x_{g}\right)$, with $k_{T}$ being the thermal mass diffusion ratio. We use the first-order approximation for $k_{T}$ provided by Chapman and Cowling [1970] and recalled in Hudson et al. [2007] who studied water vapor diffusion in the Martian subsurface environment. From equation (9), Kulmala and Vesala [1991] derived the integrated mass transfer rate with thermal mass diffusion, called here $I_{m, S F T H}\left(\mathrm{~kg} \mathrm{~s}^{-1}\right)$ :

$$
I_{m, S F T H}=-\frac{4 \pi a p M_{v} D}{R T_{\infty}}(s f+t h) .
$$

with:

$$
\begin{gathered}
s f=\ln \left(\frac{1-x_{v, a}}{1-x_{v, \infty}}\right) \\
t h=-\frac{\alpha}{2} \frac{T_{a}-T_{\infty}}{T_{\infty}}\left(x_{v, a}+x_{v, \infty}\right)
\end{gathered}
$$

[25] We call th the thermal mass diffusion term. In order to derive the thermal mass diffusion term for water droplet condensation, Kulmala and Vesala [1991] needed to assume a radial profile $x_{v}(r)$ away from the crystal surface. They used the profile coming from the integration of mass transfer with no Stefan flow. We call it the zeroth-order $x_{v}$ profile. For our purpose of investigating thermal mass diffusion upon $\mathrm{CO}_{2}$ condensation, our zeroth-order $x_{v}$ profile will need to be based on the mass transfer rate accounting for Stefan flow (see Appendix A). Thus, the term th in equation (10) will need to be replaced by $t h_{S F}$ :

$$
t h_{S F}=-\alpha \frac{T_{a}-T_{\infty}}{T_{\infty}}\left(\frac{x_{v, a}-x_{v, \infty}}{\ln \left(\frac{1-x_{v, a}}{1-x_{v, \infty}}\right)}+1\right)
$$

[26] We compare $t h_{S F}$ to the thermal mass diffusion term obtained by Kulmala and Vesala [1991], th. Figure 3 shows different plots of the ratio $t h / t h_{S F}$, in relation to $x_{v, \infty}$, for distinct fixed values of $x_{v, a}$. Since $t h / t h_{S F} \leq 1$, the Stefan flow increases the thermal mass diffusion influence on the total mass transfer rate $I_{m, S F T H}$. We also find the expected asymptotic behavior: $t h / t h_{S F} \rightarrow 1$ when $x_{v, a} \rightarrow 0$ and $x_{v, \infty} \rightarrow 0$. It is expected because in the case of a trace gas $\left(x_{v} \ll 1\right)$, the Stefan flow does not play any role in the mass transfer and $t h_{S F} \rightarrow t h$. This corresponds to the top left corner of the plot in Figure 3 where values of $t h / t h_{S F}$ are close to 1. For abundances $x_{v, \infty}$ and $x_{v, a}$ close to 1 (the right top corner of the plot in Figure 3 ), it can be seen that again $t h / t h_{S F} \sim 1$. Thus, as long as the mole fraction difference remains small, it is acceptable to use equation (12) instead of equation (13) in the case of a near-pure vapor (including the case where $x_{v, \infty}=0.75$ which is the minimum abundance value expected for $\mathrm{CO}_{2}$ ). It finally appears from Figure 3

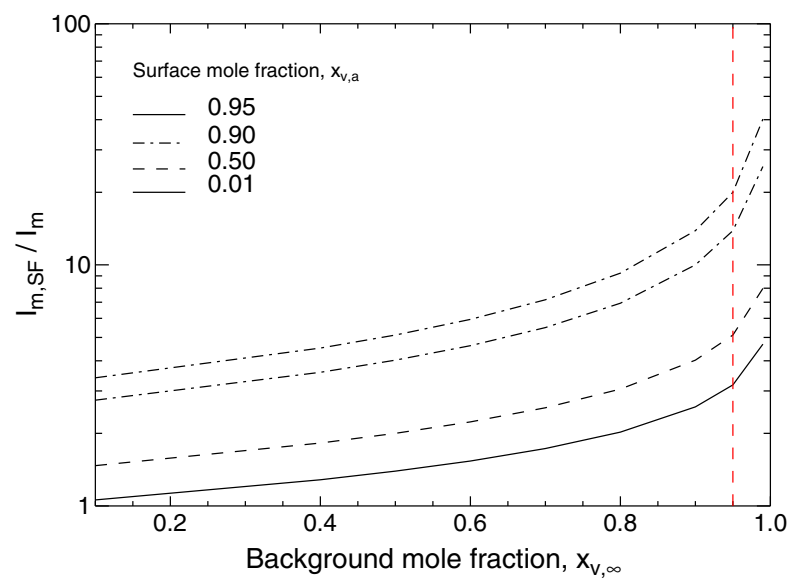

Figure 3. The ratio $t h / t h_{S F}$ is the ratio of the thermal mass diffusion term derived without and with the Stefan flow. It is plotted as a function of the background mole fraction of condensible vapor $x_{v, \infty}$, for different vapor mole fraction at the crystal surface $x_{v, a}: 0.1 \%$ (solid line), 10\% (dot line), $75 \%$ (dashed line), 90\% (dot-dashed line), and $95 \%$ (triple-dot-dashed line). The vertical dashed line matches the average $95 \% \mathrm{CO}_{2}$ present in the Martian atmosphere. The Mars conditions can be found at the top right corner of the plot where mole fractions are close to 1, whereas Earth conditions would be found at the top left corner of the plot for trace gas abundances. In both cases, $t h / t h_{S F} \sim 1$. 
that only for mole fraction difference $\left|x_{v, a}-x_{v, \infty}\right| \gtrsim 0.30$, the ratio $t h / t h_{S F}$ acquires values down to 0.70 indicating a $30 \%$ difference between both thermal mass diffusion terms. For instance, with the average $\mathrm{CO}_{2}$ mole fraction on Mars, $x_{v, \infty}=0.95$, we would have $t h / t h_{S F}<0.95$ for a much smaller surface mole fraction $\left(x_{v, a}<0.50\right)$. We will confirm in section 3.3.2 that the mole fraction difference in the present atmospheric application remains small enough to consider that $t h / t h_{S F} \sim 1$. Thus, the effect of Stefan flow in the derivation of the thermal mass diffusion term can be neglected for $\mathrm{CO}_{2}$ diffusion in the atmosphere of Mars, and we can make use of equations (10) to (12) to investigate the influence of the thermal mass diffusion.

\subsubsection{Dufour Effect}

[27] The Dufour effect is to heat transfer what thermal mass diffusion is to mass transfer. It accounts for the concentration gradient influence on the heat transfer. First we recall the equation of energy balance coupling heat and mass transfer. We do not take into account any radiative heat transfer in this study (see discussion in section 3.2). Given a mass transfer rate $I_{m}$ in spherical geometry,

$$
L_{\mathrm{sub}} I_{m}=-I_{h}=4 \pi r^{2} K \frac{\mathrm{d} T}{\mathrm{~d} r},
$$

where $L_{\text {sub }}$ is the latent heat of sublimation $\left(\mathrm{J} \mathrm{kg}^{-1}\right)$ and $r$ the distance from the crystal center. The heat released by the phase transition from vapor to ice is carried away ( $I_{h}$ from equation (5) is $<0$ ) according to the Fourier's law (righthand side term of equation (14)).

[28] The surface temperature $T_{a}$, derived by assuming $K$ and $L_{\text {sub }}$ constant and $I_{m}$ independent of $r$, is

$$
T_{a}=T_{\infty}-\frac{L_{s u b} I_{m}}{4 \pi a K}
$$

[29] This is another way to express the energy balance (equation (14)) at the crystal surface. Kulmala and Vesala [1991] showed that the Dufour effect modifies equation (14) as follows:

$$
L_{\text {sub }} I_{m}=4 \pi r^{2} K \frac{\mathrm{d} T}{\mathrm{~d} r}-\alpha R B T I_{m},
$$

where $B=\left(M_{g} x_{g}^{2} / M_{v}-x_{v} x_{g}\right) /\left(x_{v} M_{v}+x_{g} M_{g}\right)$.

[30] We notice in equation (16) a new contribution to the heat transfer, which differs from heat conduction as given by Fourier's law. This new term involves the mass transfer rate $I_{m}$, and through it the mole fraction difference. For the $\mathrm{CO}_{2} / \mathrm{N}_{2}$ gas mixture with $\mathrm{CO}_{2}$ as condensible vapor, $B$ is $<0$ and $\alpha \sim 0.4>0$ on the whole mole fraction range of $\mathrm{CO}_{2}$ encountered on Mars: typically from $x_{v} \sim 75 \%$ at the south pole [Sprague et al., 2007] to $x_{v} \sim 95 \%$. Thus, in case of condensation $\left(I_{m}<0\right)$, we have $\left(-\alpha R B T I_{m}\right)<0$. Moreover, $\mathrm{d} T / \mathrm{d} r$ has a negative sign, and then the heat conduction term $4 \pi a^{2} K(\mathrm{~d} T / \mathrm{d} r)$ too. Therefore, since heat conduction carries away from the crystal the heat released at the surface, so does the Dufour effect, which thus helps the crystal to grow. Note that $|\alpha R B T| \ll L_{\text {sub }}$ in equation (16). However, since $\left|4 \pi a^{2} K \mathrm{~d} T / \mathrm{d} r\right|$ can be as small as $\left|\alpha R B T I_{m}\right|$, the latter cannot be neglected to solve for the mass flux in equation (16). Indeed, because of high mean free path values $\lambda$ encountered in the mesosphere: $\left|4 \pi a^{2} K \mathrm{~d} T / \mathrm{d} r\right| \sim\left|4 \pi a^{2} K T_{\infty} / \lambda\right| \sim$ $\left|\alpha R B T I_{m}\right|$.
[31] The associated temperature profile, derived by assuming $K, L_{\text {sub }}$ and $B$ constant is, according to Kulmala and Vesala [1991],

$$
T_{a}=T_{\infty} \exp \left(-\frac{I_{m} \alpha R B}{4 \pi a K}\right)+\frac{L_{\text {sub }}}{\alpha R B}\left(\exp \left(-\frac{I_{m} \alpha R B}{4 \pi a K}\right)-1\right) .
$$

[32] It can be seen that if $\left|I_{m} \alpha R B /(4 \pi a K)\right| \ll 1$ then equation (17) reduces to equation (15).

[33] Equation (17) of the surface temperature accounting for the Dufour effect appears too complicated to be considered in equation (10) which includes thermal diffusion, without any explicit resolution for the mass transfer rate. However, in the case of heat release during condensational growth as considered above, $T_{a}>T_{\infty}$ so that in equation (10), the thermal mass diffusion term th is $<0$ (because $\alpha>0$ ), and thus, the thermal mass diffusion in equation (10) reduces the total mass transfer rate $I_{m, S F T H}$. It shows that thermal mass diffusion tends to lower the growth rate, in contrary to the Dufour effect, so that these effects seem to act in opposite directions.

\subsection{Coupling Mass and Heat Transfer \\ 2.3.1. Sets of Equations}

[34] We aim at deriving the growth rate of a $\mathrm{CO}_{2}$ ice crystal in the Martian atmosphere, assuming background properties are known: $p, T_{\infty}, x_{v, \infty}$ (or, equivalently, $p_{v, \infty}$ ). The unknowns of the problem are $I_{m}, T_{a}$, and $x_{v, a}$. Up to now we have only considered equations giving the mass transfer rate $I_{m}$ and the surface temperature $T_{a}$. Thus, we need a third equation for $x_{v, a}$ to ensure the closure of the system. In addition to the mass transfer rate equation and the energy balance equation that give access to the surface temperature, we add the constraint that the surface partial pressure $p_{v, a}$ equals the saturation vapor pressure of $\mathrm{CO}_{2}$ :

$$
p_{v, a}=p_{\text {sat }}\left(a, T_{a}\right)
$$

where

$$
\begin{gathered}
p_{\text {sat }}\left(a, T_{a}\right)=p_{\text {sat }}\left(\infty, T_{a}\right) \times \exp \left(\frac{2 \sigma M_{v}}{a \rho_{\mathrm{ice}} R T_{a}}\right), \\
p_{\text {sat }}\left(\infty, T_{a}\right)=p_{\text {sat }}\left(\infty, T_{\infty}\right) \exp \left(\frac{L_{\mathrm{sub}} M}{R} \frac{T_{a}-T_{\infty}}{T_{a} T_{\infty}}\right) .
\end{gathered}
$$

[35] The pressure $p_{\text {sat }}\left(\infty, T_{\infty}\right)$ is the saturation vapor pressure of $\mathrm{CO}_{2}$ over a flat surface at $T_{\infty}, \sigma$ the surface energy of $\mathrm{CO}_{2}$ ice $\left(\mathrm{J} \mathrm{m}^{-2}\right)$, and $\rho_{\text {ice }}$ the density of $\mathrm{CO}_{2}$ ice $\left(\mathrm{kg} \mathrm{m}^{-3}\right)$. The partial pressure in equation (18) is corrected for curvature effect with the Kelvin factor (exponential term in equation (19)). The Clausius-Clapeyron formula is integrated between $T_{a}$ and $T_{\infty}$ (equation (20)) assuming $\mathrm{CO}_{2}$ is an ideal gas with slight variations of $L_{\text {sub }}$ over the temperature range; $x_{v, a}=p_{v, a} / p$ so that we now have three equations for three unknowns $\left(I_{m}, x_{v, a}\right.$, and $\left.T_{a}\right)$. We can then derive the growth rate of the crystal $\mathrm{d} r / \mathrm{d} t$ through the derivation of $I_{m}$ (equation (3)).

[36] It should be noted that a crystal forming in the atmosphere falls through sedimentation which causes air motion around it. Due to this effect, a falling crystal will grow faster than a stationary crystal. The ventilation coefficient $f_{v}$ [Pruppacher and Klett, 2010] accounts for this enhancement and mainly depends on the Reynolds number. Due to very low atmospheric densities on Mars and small particle sizes, 


\section{LISTOWSKI ET AL.: $\mathrm{CO}_{2}$ ICE CRYSTAL GROWTH ON MARS}

Table 1. Presentation of the Different Growth Models Considered in This Work, the Acronyms Used, and Corresponding Equations ${ }^{\mathrm{a}}$

\begin{tabular}{lcc}
\hline Model Name & Additional Processes & Equations Considered \\
\hline CLASSIC & none & $(4),(15),(18)^{\mathrm{b}}$ \\
SF & Stefan flow & $(8),(15),(18)^{\mathrm{b}}$ \\
SFTHDUF & Stefan flow and Thermal mass diffusion/Dufour effect & $(10),(17),(18)^{\mathrm{b}}$ \\
Linearized $^{\mathrm{c}}$ & none & 24 \\
\hline
\end{tabular}

${ }^{\mathrm{a}} \mathrm{All}$ the models implicitly include the Fuchs and Sutugin correction.

${ }^{\text {b Note that }} T_{a} T_{\infty}$ is taken as $T_{\infty}^{2}$ in the Clausius-Clapeyron term (equation (20)), and that in the Kelvin factor (equation (19)), $T_{a}$ is taken as $T_{\infty}$ (see text for details)

${ }^{\mathrm{c}} \mathrm{See}$ definition of linearized model in section 3.3.1.

compared to the mean free path, this factor is very close to one and, thus, can be neglected.

[37] Table 1 presents the different models that have been investigated, each time with the processes involved and the equations used to find $I_{m}$. In the Kelvin factor, $T_{a}$ is taken as $T_{\infty}$ because it is the radius $r$ that drives its variations and not the surface temperature. Indeed for a given radius, taking $T_{\infty}$ instead of $T_{a}=T_{\infty}+\Delta T$ with $\Delta T \sim 10 \mathrm{~K}$ at most (see further section 3.3.2) does not significantly modify at all the Kelvin factor value. In the same way, in equation (20), $\left(T_{a}-T_{\infty}\right) /\left(T_{a} T_{\infty}\right)$ is taken as equal to $\left(T_{a}-T_{\infty}\right) /\left(T_{\infty}^{2}\right)$. In order to solve the equations for the CLASSIC, SF, and SFTHDUF models, we use an iterative method (NewtonRaphson method) since no explicit formulation of $I_{m}$ can be derived.

\subsubsection{Properties of the $\mathrm{CO}_{2} / \mathrm{N}_{2}$ Gas Mixture}

[38] Table 2 summarizes individual physical and thermodynamic properties we use for the $\mathrm{CO}_{2} / \mathrm{N}_{2}$ system (with their corresponding references). Note that for comparison purposes, we use the same saturation vapor pressure of $\mathrm{CO}_{2}$ as W1999 [James et al., 1992]. However, a more recent derivation of $p_{\text {sat }}(T)$ for $\mathrm{CO}_{2}$ has been done by Azreg-Ä̈nou [2005] and showed a perfect match with James et al. [1992]. This is important because saturation vapor pressure has a strong dependency with temperature and thus is a critical parameter for the growth rate. In the following, we discuss the transport properties of the system.

\subsubsection{Diffusion Coefficient $D_{\mathrm{CO}_{2} / \mathbf{N}_{2}}$}

[39] C2003 used for the diffusion coefficient $D$ the selfdiffusion coefficients of $\mathrm{CO}_{2}$ an $\mathrm{N}_{2}$ weighted by $x_{v}$ and $x_{g}$ (see C2003, equation (13)). Since we could not find in the literature a theoretical justification for this approach, we have proceeded in a different way. For liquid mixture, binary diffusion coefficient can indeed be very composition dependent as recalled by Poling et al. [2001, section 11.7] but for gas mixtures, dependencies on mole fractions for $D$ can occur only with three or more components (see, e.g., Mathur and Saxena [1966] for successful application to ternary cases). In a binary gas mixture, only the binary diffusion coefficient as presented in equation (21) should be used. We thus use a diffusion coefficient that suits to binary gas systems at low pressures [Fuller et al., 1966] mentioned in Poling et al. [2001] as one of the most general and reliable:

$$
D_{\mathrm{CO}_{2} / \mathrm{N}_{2}}=\frac{0.00143 T^{1.75}}{P M_{\mathrm{CO}_{2}, \mathrm{~N}_{2}}^{1 / 2}\left[\left(\Sigma_{v}\right)_{\mathrm{CO}_{2}}^{1 / 3}+\left(\Sigma_{v}\right)_{\mathrm{N}_{2}}^{1 / 3}\right]^{2}}
$$

where $D_{\mathrm{CO}_{2} / \mathrm{N}_{2}}$ is in $\mathrm{cm}^{2} \mathrm{~s}^{-1}, T$ is the temperature $(\mathrm{K}), P$ is the pressure in bar, $M_{\mathrm{CO}_{2}, \mathrm{~N}_{2}}=\left(1 / M_{\mathrm{CO}_{2}}+1 / M_{\mathrm{N}_{2}}\right)^{-1}$ is in $\mathrm{g} \mathrm{mol}^{-1}$, and $\Sigma_{v}$ is the atomic diffusion volumes given in Fuller et al. [1969].

\subsubsection{Thermal Conductivities}

[40] The most recent reference studies about carbon dioxide thermal conductivity are Vesovic et al. [1990] and Scalabrin et al. [2006], for temperature higher than $200 \mathrm{~K}$.

Table 2. Physical and Thermodynamic Properties of $\mathrm{CO}_{2}$ and $\mathrm{N}_{2}$

\begin{tabular}{|c|c|c|c|c|}
\hline Variable & Name & Unit & Value & Reference \\
\hline$M_{\mathrm{CO}_{2}}$ & molar mass & $\mathrm{g} \mathrm{mol}^{-1}$ & 44.01 & Span and Wagner [1996] \\
\hline$\rho_{c}$ & critical density & $\mathrm{kg} \mathrm{m}^{-3}$ & 467.6 & Span and Wagner [1996] \\
\hline$\rho_{\text {ice }}$ & ice density & $\mathrm{kg} \mathrm{m}^{-3}$ & 1600.0 & $\operatorname{Wood}[1999]$ \\
\hline$\sigma$ & surface energy & $\mathrm{J} \mathrm{m}^{-2}$ & 0.080 & Wood [1999] \\
\hline$L_{\text {sub }}(T)$ & latent heat of sublimation & $\mathrm{J} \mathrm{kg}^{-1}$ & $\begin{array}{c}\sum_{i=0}^{4} l_{i} T^{i} \\
l_{0}=595594 \\
l_{1}=903.111 \\
l_{2}=-11.5959 \\
l_{3}=0.0528288 \\
l_{4}=1.03183 \times 10^{-4}\end{array}$ & Azreg-Ä̈nou $[2005]^{\mathrm{a}}$ \\
\hline$p_{\text {sat }}(T)$ & saturation vapor pressure & $\mathrm{Pa}$ & $\begin{array}{l}A \exp (-B / T) \\
A=1.382 \times 10^{12} \mathrm{~Pa} \\
B=3182.48 \mathrm{~K}\end{array}$ & James et al. $[1992]^{\mathrm{b}}$ \\
\hline$k_{v}$ & thermal conductivity & $\mathrm{W} \mathrm{m} \mathrm{m}^{-1} \mathrm{~K}^{-1}$ & see equation (17) of reference & Scalabrin et al. [2006] \\
\hline$M_{\mathrm{N}_{2}}$ & molar mass & $\mathrm{g} \mathrm{mol}^{-1}$ & 28.01 & Lemmon and Jacobsen [2004] \\
\hline$\rho_{c}$ & critical density & $\mathrm{kg} \mathrm{m}^{-3}$ & 313.15 & Lemmon and Jacobsen [2004] \\
\hline$k_{g}$ & thermal conductivity & $\mathrm{W} \mathrm{m} \mathrm{m}^{-1} \mathrm{~K}^{-1}$ & see equation (4) of reference & Lemmon and Jacobsen [2004] \\
\hline
\end{tabular}

${ }^{a}$ Formula obtained from a fourth-order polynomial fit of the data presented in the reference paper.

${ }^{\mathrm{b}}$ We chose the same expression rather than Wood [1999]. A most recent and independent derivation of saturation vapor pressure by Azreg-Ä̈nou [2005] has shown a perfect match with the one of James et al. [1992] 


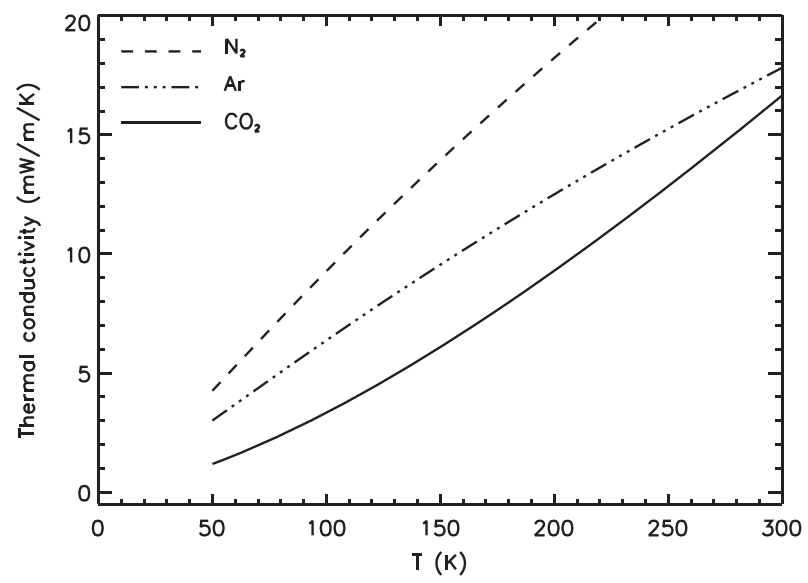

Figure 4. Thermal conductivities in $\mathrm{mW} \mathrm{m}^{-1} \mathrm{~K}^{-1}$ plotted against temperature for $\mathrm{N}_{2}$ (dotted line) and $\mathrm{Ar}$ (triple-dotdashed line) as given by Lemmon and Jacobsen [2004] and $\mathrm{CO}_{2}$ as given by Scalabrin et al. [2006] (thick line). The $\mathrm{CO}_{2}$ thermal conductivity is extrapolated below $\sim 200 \mathrm{~K}$.

Scalabrin et al. [2006] only used measurements and their correlation for $\mathrm{CO}_{2}$ thermal conductivity matches the one of Vesovic et al. [1990] which also includes theoretical considerations valid for the temperature range investigated $(T \geq$ $200 \mathrm{~K})$. We extrapolate Scalabrin et al.'s [2006] correlation below $200 \mathrm{~K}$ and obtain the plot showed in Figure 4. As stated in Poling et al. [2001], "thermal conductivities of low-pressure gases increase with temperature," which we do observe for the extrapolation of $\mathrm{CO}_{2}$ thermal conductivity. We also show for comparison the variations with temperature of the $\mathrm{N}_{2}$ and Argon (Ar) thermal conductivities. Quantities are plotted for a density of $\rho=0.01 \mathrm{~kg}$ $\mathrm{m}^{-3}$ (typical of the Martian surface). However, for typical current Martian densities the dependence of thermal conductivities on density is negligible. An appreciable difference only arises when $\rho \gtrsim 10 \mathrm{~kg} \mathrm{~m}^{-3}$, i.e., when density reaches roughly $1 / 30$ of the critical densities $\rho_{c}$ (Table 2).

[41] For nitrogen $\mathrm{N}_{2}$ and Ar, Lemmon and Jacobsen [2004] present a thermal conductivity correlation valid for all pressure and temperature domains. Argon is the third most abundant gas in the Martian atmosphere $(\mathrm{Ar} \sim 1.6 \%)$ after nitrogen $\left(\mathrm{N}_{2} \sim 2.7 \%\right)$. At this point we can say that the inert $5 \%$ of the Martian atmosphere can be assumed to be exclusively composed of $\mathrm{N}_{2}$, since the $\mathrm{N}_{2}$ and $\mathrm{Ar}$ thermal conductivities are of the same order of magnitude. Molecules with more than one atom are susceptible to have contributions of internal degrees of freedom (rotational and vibrational) in their thermal conductivity. However, the trends of $\mathrm{Ar}$ and $\mathrm{N}_{2}$ thermal conductivity toward low temperatures at low Martian pressures are the same: a smooth decrease with temperature. $\mathrm{CO}_{2}$ being a triatomic, linear, and nonpolar molecule, we can assume that a behavior similar to $\mathrm{N}_{2}$ (as $\mathrm{N}_{2}$ is similar to $\mathrm{Ar}$ ) is consistent.

\subsubsection{Thermal Conductivity of the Mixture}

[42] Since both the condensible vapor and the inert gas contribute to the evacuation of the latent heat away from the growing crystal, it appears more relevant to include the influence of relative abundances on transport processes in the thermal conductivity rather than in the diffusion, as previously discussed. We use the thermal conductivity of the vapor-gas mixture, relying on the empirical relation first proposed by Wassiljeva [1904], as suggested by Poling et al. [2001] for nonpolar gas mixture,

$$
K=\frac{x_{v}}{x_{v} A_{v v}+x_{g} A_{v g}} k_{v}+\frac{x_{g}}{x_{v} A_{g v}+x_{g} A_{g g}} k_{g}
$$

where $K, k_{v}$, and $k_{g}$ are, respectively, the thermal conductivity of the mixture, of the condensible vapor $\left(\mathrm{CO}_{2}\right)$, and of the inert gas $\left(\mathrm{N}_{2}\right)$ in $\mathrm{W} \mathrm{m}^{-1} \mathrm{~K}^{-1} . A_{v v}, A_{g g}, A_{v g}, A_{g v}$ are coefficients given by equation (10-6.2) in Poling et al. [2001].

\section{Results}

\subsection{The Relative Importance of the Different Processes on the Growth Rate}

[43] In order to compare the impact of the different processes described in section 2.2 within Martian atmospheric conditions we define the growth rates related to the models summarized in Table 1: $(\mathrm{d} r / \mathrm{d} t)_{\text {CLASSIC }},(\mathrm{d} r / \mathrm{d} t)_{S F}$ and $(\mathrm{d} r / \mathrm{d} t)_{\text {SFTHDUF }}$, derived as explained in section 2.3.1.

[44] Figure 5 shows ratios of the growth rates plotted versus atmospheric pressure $p$. We compare each time $(\mathrm{d} r / \mathrm{d} t)_{S F}$ or $(\mathrm{d} r / \mathrm{d} t)_{\text {SFTHDUF }}$ to $(\mathrm{d} r / \mathrm{d} t)_{\text {CLASSIC }}$, in order to evaluate the relative importance of Stefan flow and thermal mass diffusion/Dufour effect on the growth rate in Martian atmospheric conditions. We examine the growth rates over a large pressure range $\left(10^{-3}-5.10^{5} \mathrm{~Pa}\right)$, which includes the typical pressure range of the Martian atmosphere: from $\sim 600 \mathrm{~Pa}$ (at the surface) to $\sim 0.001 \mathrm{~Pa}$ in the mesosphere $(\sim 100 \mathrm{~km})$. We stop at $p \sim 5.10^{5} \mathrm{~Pa}$ because there lies the triple point pressure of $\mathrm{CO}_{2}\left(p_{t}=5.185 \times 10^{5} \mathrm{~Pa}\right)$, above which all three phases, vapor, liquid, and ice, can appear. Moreover, ratios are plotted over a large range of saturation ratios going up

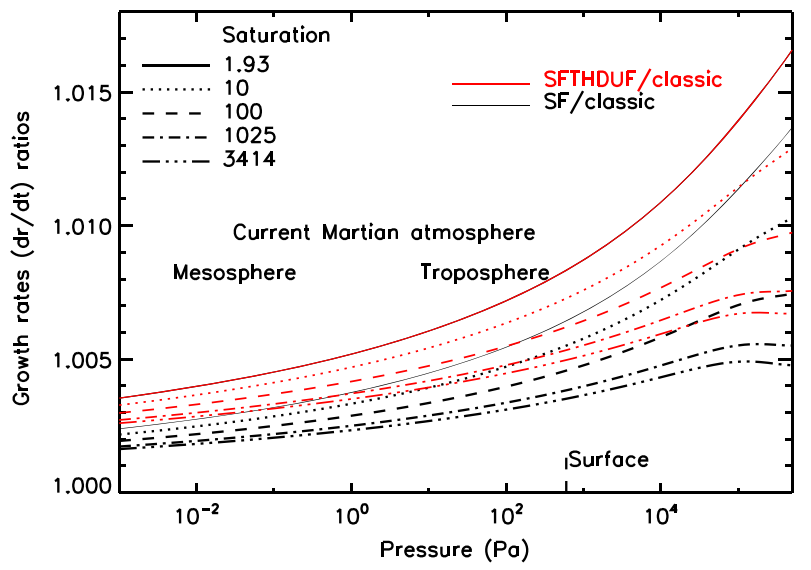

Figure 5. Growth rate ratios $(\mathrm{d} r / \mathrm{d} t)_{\text {SFTHDUF }} /(\mathrm{d} r / \mathrm{d} t)_{\text {CLASSIC }}$ (red lines) and $(\mathrm{d} r / \mathrm{d} t)_{S F} /(\mathrm{d} r / \mathrm{d} t)_{\text {CLASSIC }}$ (black lines) are plotted for a particle radius $r=10 \mathrm{~nm}$ as a function of the atmospheric pressure $P$ going from $0.001 \mathrm{~Pa}$ (Martian high mesosphere) to $5.10^{5} \mathrm{~Pa}$ (triple point pressure of $\mathrm{CO}_{2}$ ). "Surface" indicates the mean pressure at Mars' surface. Different saturation ratios are considered, going from $S \sim 2$ to $S \sim 3400$. The three models CLASSIC, SF, and SFTHDUF are defined in Table 1. The typical Martian surface pressure is indicated at $\sim 600 \mathrm{~Pa}$. The minimum saturation ratio $(S=1.93)$ is close to the critical saturation ratio needed to overcome the Kelvin barrier for a $10 \mathrm{~nm}$ particle. 
to the order of magnitudes found by Forget et al. [2009] and Montmessin et al. [2011], i.e., $S>1000$. The minimum saturation ratio considered in Figures 5 is 1.93. This is due to the curvature effect that acts on the particles $\lesssim 100 \mathrm{~nm}$ by increasing the equilibrium saturation ratio $\left(S_{e q}>1\right)$ that ambient saturation has to overcome $\left(S\right.$ must be $>S_{\text {eq }}$ ) to allow the crystal to grow. We thus start from the first $S$ showing significant growth.

[45] Figure 5 shows that within current Martian conditions $(P \leq 600 \mathrm{~Pa})$, for a $10 \mathrm{~nm}$ crystal, the Stefan flow alone (black lines) or the Stefan flow with thermal mass diffusion and Dufour effect (red lines) will increase the growth rate at most by $\sim 1 \%$ whatever the saturation ratio (or, equivalently, the background temperature). Toward higher pressure and until $p=p_{t}$, both growth rates ratios increase. The growth rate $(\mathrm{d} r / \mathrm{d} t)_{S F}$ alone becomes $\sim 1.4 \%$ bigger than $(\mathrm{d} r / \mathrm{d} t)_{\text {CLASSIC }}$ and $(\mathrm{d} r / \mathrm{d} t)_{\text {SFTHDUF }} \sim 1.7 \%$ bigger than $(\mathrm{d} r / \mathrm{d} t)_{\text {CLASSIC. }}$ Even for bigger crystals (up to radii $\sim 100 \mu \mathrm{m}$, the largest particles expected on Mars), the behavior is qualitatively and quantitatively the same (not shown) up to $p=p_{t}$.

[46] The mass transfer rate equation, without a coupled heat transfer equation, suggested a dramatic rise of the growth rate through the Stefan flow (see Figure 2 in section 2). Heat transfer actually prevents it from happening. Heat transfer plays a major role in the energy balance by "neutralizing" the Stefan flow and the Dufour effect/thermal mass diffusion which enhance the mass transfer rate by only $\sim 1-2 \%$. Note that the lowest saturation ratio $(S=$ 1.93 , black solid curve) gives the largest (but still small) relative difference $(\sim 1.4 \%)$ of the SF model compared to the CLASSIC model, while the biggest saturation ratio $(S \sim$ 3000 , black triple-dot-dashed line) gives the lowest relative difference $(\sim 0.5 \%)$. This is because a lower temperature is needed to reach a higher saturation ratio, and thus the thermal conductivity gets lower and increases the limiting effect of heat transfer, reducing the enhancement of the mass transfer rate by the Stefan flow. Heat transfer has also a limiting effect for denser atmospheres $(P \geq 600 \mathrm{~Pa})$. This conclusion stands also for molar fraction $x_{v, \infty}<0.95$ and in particular for $x_{v, \infty} \sim 0.75$ which can be reached in the polar night atmosphere during widespread $\mathrm{CO}_{2}$ condensation [Sprague et al., 2007].

[47] Note that for $x_{v, \infty}=0.95$, it is only when artificially multiplying the thermal conductivity by $\sim 100$ that the Stefan flow becomes increasingly important for higher and higher pressures (not shown). It causes $\mathrm{a} \sim 100 \%$ enhancement of the growth rate at pressures $p \gtrsim 1-10 \mathrm{~Pa}$ (depending on the particle size). Already for a tenfold thermal conductivity, Stefan flow produces growth rates $\sim 10 \%$ bigger and more, for $p \gtrsim 10^{4} \mathrm{~Pa}$. It is thus rather for typical liquid thermal conductivities (10 to 100 times higher than typical values for gases) that Stefan flow cannot be neglected, whatever the particle radius. In this case Dufour effect and thermal mass conductivity are also negligible: They produce much slighter changes $(\sim 1 \%)$ in the growth rates than the prominent Stefan flow $(\sim 100 \%)$.

[48] Finally for current Martian conditions, Stefan flow, thermal mass diffusion, and Dufour effect can be neglected when modeling the growth of $\mathrm{CO}_{2}$ ice crystals, within the broad diversity of Martian atmospheric conditions and particle sizes. This conclusion stands as well for an ancient Mars with a $10^{5} \mathrm{~Pa}$ atmosphere since we investigated pressures as high as $5.10^{5} \mathrm{~Pa}$ and whatever the abundances encountered. Condensation rates of $\mathrm{CO}_{2}$ ice crystals can be calculated using the CLASSIC model (see Table 1), for mesospheric $\mathrm{CO}_{2}$ ice clouds as well as for polar $\mathrm{CO}_{2}$ ice clouds in the current Martian atmosphere. Evaporation rates are derived exactly in the same manner with $I_{m}$ being $>0$. The CLASSIC model becomes from now on our reference model.

\subsection{Surface Kinetics and Radiative Heat Transfer}

[49] Surface kinetics effects are set to zero, meaning that we do not account for the efficiency of vapor molecules integration into the crystal lattice. We refer the reader to W1999 for detailed explanations and discussions about different surface kinetics processes and their potential effect on the growth of $\mathrm{CO}_{2}$ ice crystals in the Martian environment. Note that W1999 argued that surface kinetics was not likely to be a limiting or significant process for evaporation, even if it were for condensation. Importantly, relying on Glandorf et al.'s [2002] experiments, C2003 noted that no particular resistance to growth due to kinetic barriers was observed for typical Martian polar conditions for $\mathrm{CO}_{2}$ condensation at $S \sim 1.35(p \sim 100 \mathrm{~Pa}, T \sim 140 \mathrm{~K})$. Even though it is not known whether such barriers could be of importance for very high saturation ratios in the upper atmosphere we assume, in line with the experimental results, that surface kinetics can be neglected.

[50] The radiative term is set to zero in order to compare our results with those of C2003 and W1999 neglecting it as well. It is not the scope of this paper to consider the effect of radiative heat transfer on crystal growth. However, it is an aspect that we will address in a future study within a complete one-dimensional model of mesospheric $\mathrm{CO}_{2}$ ice clouds (ongoing work), since radiative heat transfer may indeed play a role in crystal growth. It has been recently demonstrated that radiative heat transfer needs to be taken into account in the case of mesospheric water ice clouds on Earth [Espy and Jutt, 2002; Rapp and Thomas, 2006]. $\mathrm{CO}_{2}$ ice crystals are poorer absorbers than water ice crystals in the visible and the infrared, except for a few strong absorption bands. According to our estimations (not shown) and Wood [1999], radiative effects, in particular radiative cooling, are negligible in the polar night for tropospheric clouds. However, at increasing altitudes, where $K n \gg 1$, heat conduction becomes more and more kinetically limited, and thus the radiative cooling progressively competes with it. For the sake of comparison with C2003 and W1999, who did not include radiative effects, we have omitted to include radiative exchanges in the energy budget of the crystal.

\subsection{Comparison With Previous Studies}

\subsubsection{Growth Rate Models for Earth Clouds: Linearization of the Saturation Vapor Pressure Equation}

[51] Condensational growth formulae for water droplets on Earth as derived by Barkstrom [1978] or for water ice crystals as presented by Kuroda [1984] rely almost on the same set of equations as the CLASSIC model. No processes like Stefan flow or thermal mass diffusion and Dufour effect are considered. However, there is an important difference between Barkstrom [1978] or Kuroda [1984] and our CLASSIC model. Indeed, the former two use a linearization 


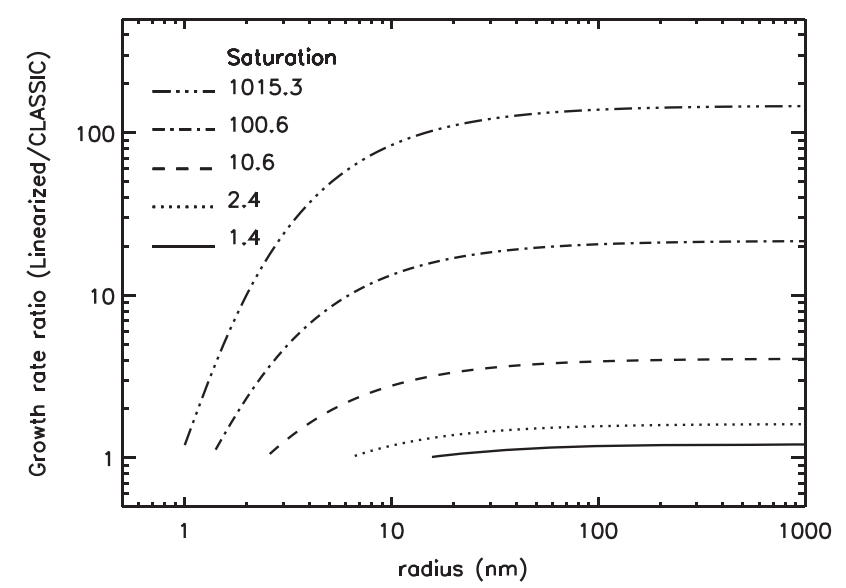

Figure 6. Ratio of the linearized model growth rate $(\mathrm{d} r / \mathrm{d} t)_{\text {Linearized }}$ (equation (24)) to the CLASSIC model growth rate $(\mathrm{d} r / \mathrm{d} t)_{\text {CLASSIC }}$ (Table 1$)$ as a function of the particle radius, for different saturation ratios $S: 1.4$ (thin line), 2.4 (dotted line), 10.6 (dashed line), 100.6 (dot-dashed line), and 1015.3 (triple-dot-dashed line). The curves do not start from the same radius due to the Kelvin barrier.

of the saturation vapor pressure temperature dependance in order to get an explicit formulation of the growth rate $\mathrm{d} r / \mathrm{d} t$. This approximation consists in linearizing the exponential term of equation (20) in the surface partial pressure $p_{v, a}$ by assuming $\left(T_{a}-T_{\infty}\right)$ is small enough (the system being close to equilibrium), so that

$$
\exp \left(\frac{L_{\mathrm{sub}} M}{R} \frac{T_{a}-T_{\infty}}{T_{a} T_{\infty}}\right) \sim 1+\frac{L_{\mathrm{sub}} M}{R} \frac{T_{a}-T_{\infty}}{T_{a} T_{\infty}}
$$

If we proceed in the same way and expand to the first order the exponential term in our CLASSIC model, we get, after some algebra, the following growth rate:

$$
\frac{\mathrm{d} r}{\mathrm{~d} t}=\frac{1}{r} \frac{S-S_{e q}}{R_{d}+R_{h} \times S_{e q}},
$$

where $R_{d}=\rho_{\text {ice }} R T /\left(M_{v} D p_{\text {sat }}(T)\right), R_{h}=\rho_{\text {ice }} M_{v} L_{\text {sub }}^{2} /\left(K R T^{2}\right)$, and $S_{e q}=\exp \left(2 \sigma M_{v} / \rho_{\text {ice }} R T r\right)$. $S_{e q}$ is the equilibrium saturation ratio taking into account the droplet curvature through the Kelvin factor, and $r$ is the crystal radius. Following Kuroda [1984], $R_{d}$ and $R_{h}$ are called resistances by analogy with electrokinetics. $R_{d}$ and $R_{h}$ quantify the resistance to growth due to diffusion and heat conduction, respectively. The growth model that uses equation (24) will be called the "linearized model," as presented in Table 1.

[52] C2003 based their studies on early Mars $\mathrm{CO}_{2}$ ice clouds on Toon et al. [1989], which belongs to the category of linearized models. Colaprete et al. [2003] and Colaprete et al. [2008] used the same growth rate model for subsequent studies on present Mars, respectively, for south polar clouds and polar/mesospheric clouds. Tobie et al. [2003] also used a linearized growth rate [MacKenzie and Haynes, 1992] to model polar wave $\mathrm{CO}_{2}$ clouds on Mars. We show in Appendix B various expressions of linearized model growth rates and indicate whether they have been applied to model the growth of crystals on Mars.

\subsubsection{Discrepancies Between the CLASSIC Model and the Linearized Model}

[53] We compare growth rates obtained with the linearized model (equation (24)) with growth rates obtained with our CLASSIC model (Table 1). In Figure 6, the ratio $(\mathrm{d} r / \mathrm{d} t)_{\text {Linearized }} /(\mathrm{d} r / \mathrm{d} t)_{\text {CLASSIC }}$ is plotted as a function of the particle radius and for different saturation ratios. Atmospheric pressure is $p \sim 0.01 \mathrm{~Pa}$ (mesospheric conditions). Growth rates of the linearized model are from 1.2 to 150 times bigger than CLASSIC model growth rates, for $S=$ 1.4-1000. We refer the reader to section 3.3.4 to get an idea of the absolute values and relevant units of $\mathrm{d} r / \mathrm{d} t . \mathrm{CO}_{2}$ heterogeneous ice nucleation can occur already for saturation ratios as low as $S \gtrsim 1.35$ on Mars [Glandorf et al., 2002]. Thus, a difference in the $\mathrm{CO}_{2}$ ice crystal growth rate already arises for the lowest saturation ratio values of condensation onset after nucleation has taken place.

[54] The linearized model gives different (bigger) growth rates because the approximation assumed in equation (23) does not hold for a near-pure vapor condensing in highly supersaturated conditions. With increasing radius and saturation ratios, the growth rates derived from the linearized model strongly deviate from their corresponding CLASSIC value. In order to explain why we consider equation (23), the coefficient $L_{\text {sub }} M / R$ has a typical value of $\sim 3 \times 10^{3} \mathrm{~K}$ and $T_{a} T_{\infty} \sim T_{\infty}^{2} \sim 10^{4} \mathrm{~K}^{2}$. Thus, it appears that for $\Delta T \gtrsim 1 \mathrm{~K}$, it is not a valid approximation to replace the exponential factor (23) by its first-order expression. Second, in Figure 7 we show the corresponding $\Delta T$ found with the CLASSIC model for the cases Figure 6 previously discussed. Whatever the saturation ratio, the temperature difference exceeds one Kelvin, for a given radius. Thus, for a near-pure vapor like $\mathrm{CO}_{2}$ on Mars, $\Delta T$ cannot be considered as "small enough" to justify the linearization. The linearization of the exponential function in (18) results in a strong underestimation of the surface partial pressure $p_{v, a}$ so that the partial pressure difference $\left|p_{v, a}-p_{v, \infty}\right|$ is overestimated compared to what it would be without the approximation. It results that the mass transfer rate with the linearized model is higher than with the CLASSIC model. Indeed, the nonlinearized exponential factor will give a higher value of $p_{v, a}$, thus a lower partial pressure difference, and a lower ice crystal growth rate. The

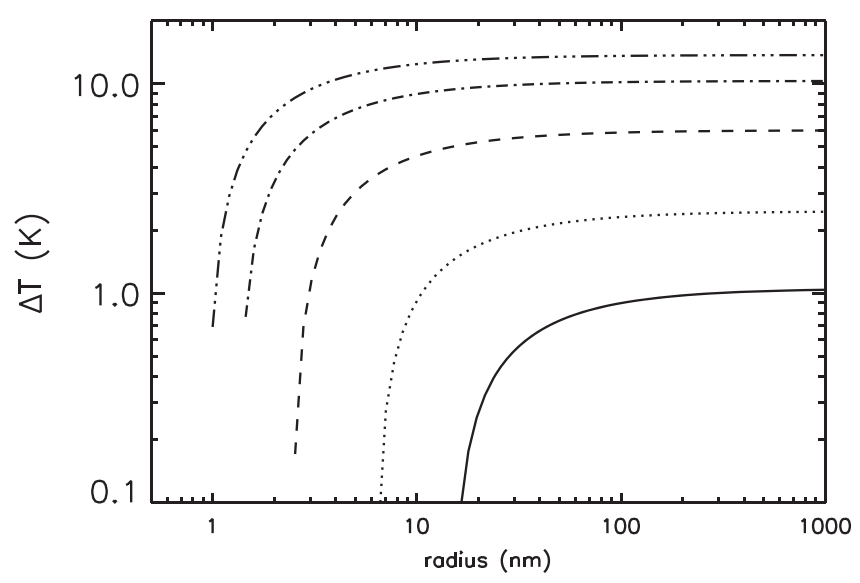

Figure 7. Temperature difference $\Delta T=T_{a}-T_{\infty}$ related to the CLASSIC growth rates derived for Figure 6 in relation to the particle radius. The curves are defined as in Figure 6. 
same logic applies for evaporation rates and the conclusion is opposite. The linearization of the exponential still results in an underestimation of $p_{v, a}$. However, in the case of evaporation, $p_{v, a}>p_{v, \infty}$ so the partial pressure gradient and the evaporation rate will be underestimated. Overall, it is interesting to see that in case of high supersaturations, the proper use of a nonlinearized expression of the surface partial pressure leads to crystal growing slower and evaporating faster than with the linearized growth/evaporation rate.

[55] In section 2.2.3.1 we made the assumption that the mole fraction differences in our atmospheric application could be considered as small enough $\left(\left|\Delta x_{v}\right| \ll 1\right)$, in order to use the thermal mass diffusion expression which ignores the Stefan flow contribution (equation (12)). We find that, indeed, the mole fraction differences are on the order of $10^{-5} \lesssim\left|x_{v, a}-x_{v, \infty}\right| \lesssim 10^{-2}$ and thus validate the assumption of section 2.2.3.1.

[56] The linearized model is suitable to water ice cloud crystals in Martian conditions [Michelangeli et al., 1993; Colaprete et al., 1999; Pathak et al., 2008; Daerden et al., 2010; Montmessin et al., 2002, 2004] (see Table 1) because water vapor is a trace gas on Mars and related temperature differences should remain low enough for the linearization to hold. Indeed, the mass transfer rate $I_{m}$ for a trace gas is lower than in the near-pure vapor case (smaller mole fraction difference) so that $\Delta T=-L_{\text {sub }} I_{m} /(4 \pi a K)$ would remain small enough (equation (15)). However, using a linearized model for $\mathrm{CO}_{2}$ ice clouds on Mars is not appropriate if we are interested in the mesosphere where saturation ratios can reach extreme values, going up to $S \sim 1000$ which corresponds to $\Delta T \sim 10 \mathrm{~K}$ (Figure 7). The mass transfer rate $I_{m}$ in a near-pure vapor case with high supersaturations, higher than in the trace gas case, and the kinetically limited regime because of the rarefied atmosphere create and maintain a high temperature difference.

\subsubsection{Comments on Colaprete and Toon's [2003] Approach for $\mathrm{CO}_{2}$ Condensation}

[57] In this section we discuss the applicability of Toon et al.'s [1989] model to Martian $\mathrm{CO}_{2}$ clouds, which is well known in the terrestrial community. In the most recent review on the microphysical modeling of the Polar Mesospheric Clouds (PMCs) on Earth [Rapp and Thomas, 2006], the authors mention the use of Toon et al. [1989] to model the growth of the water ice crystals in Earth mesospheric conditions. PMCs are known to be the closest counterpart to Martian mesospheric clouds [Määttänen et al., 2012]. Colaprete and Toon [2003] applied the model of Toon et al. [1989] to Martian $\mathrm{CO}_{2}$ clouds in ancient Mars. This model was further used by Colaprete et al. [2008] to model polar and mesospheric clouds.

[58] The growth rate used by Toon et al. [1989] differs slightly from the other linearized models. Toon et al. [1989] indicate that their growth rate model for polar stratospheric ice crystals comes from the work of Barkstrom [1978] and Ramaswamy and Detwiler [1986]. However, their formula differs from equation (24) which does correspond to the one used by Barkstrom [1978] or Ramaswamy and Detwiler [1986] without the radiative term. With our notation, Toon et al.'s [1989] growth rate formula can be expressed as follows (see third line of Table B1 as well):

$$
\frac{\mathrm{d} r}{\mathrm{~d} t}=\frac{1}{r} \frac{S-S_{e q}}{R_{d}+R_{h} \times S} .
$$

[59] Note that the term $R_{h} \times S_{\text {eq }}$ of equation (24) has been here replaced by $R_{h} \times S$ (in Toon et al.'s [1989] work, $S$ is expressed as a ratio of number densities $n_{\infty} / n_{\text {sat }}$ - see their appendix). One explanation for this formulation could be that for the low saturation ratios and particle sizes encountered on Earth in the polar stratosphere $S \sim S e q$, so that equation (25) is equivalent to equation (24). Nevertheless, this $S$ factor at the denominator does not come from the linearization of the saturation vapor pressure temperature dependance only.

[60] If we apply equation (25) as it stands in C2003, to the Martian conditions where $S$ can be $\gg S_{e q}$, growth rates appear much lower than the CLASSIC ones. We found that the growth rates $(\mathrm{d} r / \mathrm{d} t)_{\text {Toon }}$ are lower for saturation ratios $S \gtrsim 1.4$, compared to $(\mathrm{d} r / \mathrm{d} t)_{\text {CLASSIC }}$ (not shown); $(\mathrm{d} r / \mathrm{d} t)_{\text {Toon }}$ is $16 \%$ to $86 \%$ lower than $(\mathrm{d} r / \mathrm{d} t)_{\text {CLASSIC }}$, respectively, for the saturation ratios in the range $S=1.4-1000$. Thus, this growth rate would be inappropriate for large saturations ratios as the one encountered in the mesosphere.

[61] We now focus on saturation ratios $S>1$ as recently derived by $\mathrm{Hu}$ et al. [2012] in polar regions ( $p \sim 500$ $40 \mathrm{~Pa}$ ) using the radio occultations from RS/MGS and the climate sounder MCS/MRO. The authors reported mean saturation ratio in both hemispheres around $1.25-1.3$ so that using equation (25) or equation (24) with these conditions would not cause a difference of more than $10 \%$ in the growth rates, compared to the CLASSIC model. Thus, using a linearized model to derive the $\mathrm{CO}_{2}$ ice crystal growth rates in typical polar conditions $(S=1.25-1.3)$ as reported by $H u$ et al. [2012] should not lead to appreciable errors.

[62] Nevertheless, Hu et al. [2012] reported also maximum saturation ratios of 2 and 2.2 in the northern polar regions and the southern polar regions, respectively. We now choose an average polar pressure at condensation level of $\sim 80 \mathrm{~Pa}$ (as we use it for comparison purposes with W1999's work in polar conditions_-see section 3.3.4). Using the linearized model (equation (24)) for radii $r \geq$ $100 \mathrm{~nm}$, we find $\sim 15 \%-40 \%$ bigger growth rates than with our CLASSIC model, for $S=1.4-2.4$. Conversely, using equation (25), $\mathrm{d} r / \mathrm{d} t_{\text {Toon }}$ is respectively $13 \%$ to $25 \%$ lower than $(\mathrm{d} r / \mathrm{d} t)_{\text {CLASSIC. If such saturation ratios as the maximum }}$ $S=2.2$ observed by Hu et al. [2012] occur in the polar cloud environment, it might be advisable to test the difference of linearized versus nonlinearized (CLASSIC) growth rates on the cloud properties.

\subsubsection{Comparison to Wood's [1999] Approach for $\mathrm{CO}_{2}$ Condensation}

[63] In this section we compare our CLASSIC model with W1999 model which relies on equations developed by Young [1993, hereafter Y93]. W1999 investigated the formation of polar snow on Mars at tropospheric altitudes. W1999 used the most complete set of equations, to our knowledge, for condensational growth with the same saturation vapor pressure of $\mathrm{CO}_{2}$ [James et al., 1992]. It consists of a condensation model [Young, 1991, 1993], for a binary gas mixture, valid for any Knudsen number and any relative abundance of vapor (from $x_{v} \ll 1$ to $x_{v}=1$ ). Y93 uses a "Langmuir-type" model (see section 2.1) to derive growth (evaporation) rates. While Y93 applies kinetic physics with appropriate velocity distribution functions in the Knudsen layer, outside of the Knudsen layer, the author used the conservation equations of continuum physics in the infinite reservoir of vapor-gas 


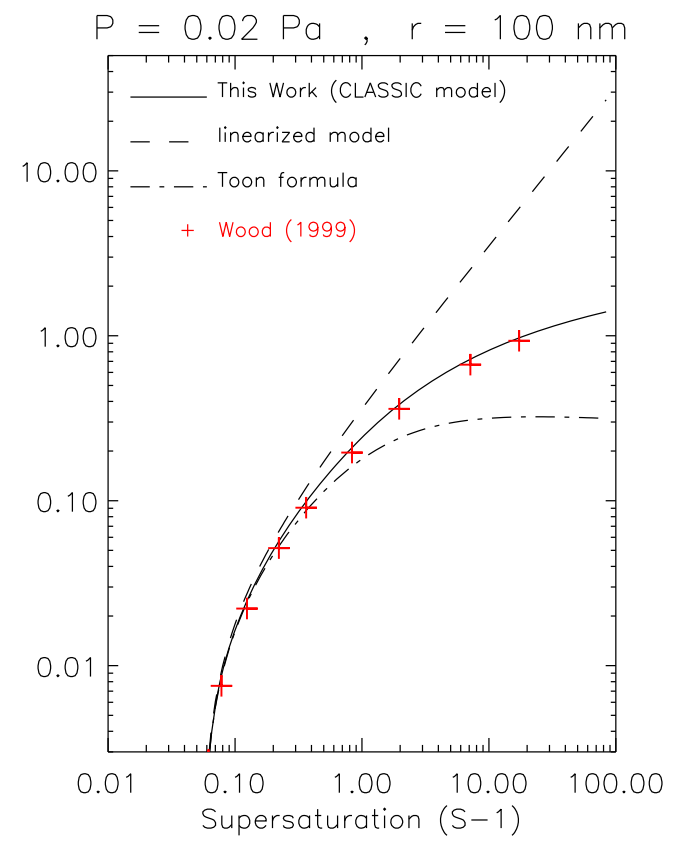

Figure 8. Growth rate in $\mu \mathrm{m} \mathrm{h}^{-1}$ plotted against supersaturation $(S-1)$ for a mesospheric pressure $p=0.02 \mathrm{~Pa}$ and a particle radius $r=100 \mathrm{~nm}$, at $x_{v, \infty}=95 \%$. Dashed line is the linearized model as given by equation (24), and the solid line is the CLASSIC model developed in this work (Table 1). The dot-dashed line is the growth rate used by Colaprete and Toon [2003] (equation (25)), and initially presented in Toon et al. [1989]. Red crosses data are extracted from W1999's work (Chapter 8, Figure 8.10).

mixture. Fluxes are matched at the interface of the Knudsen layer with a specific velocity distribution accounting for the nonequilibrium state at the interface. Thus, the resulting phenomenological equations that Y93 derived account for the different regimes, from kinetic to continuum regime, passing by a transition regime. In addition to that, they include the influence of temperature gradient on the mass transfer and the influence of the partial pressure gradient on the heat transfer (the Dufour effect). Stefan flow is also taken into account by the model in the derivations of the continuum mass transfer equation (see equation (13) of Young [1993], which gives the same mass transfer rate as our equation (8) for $I_{m, S F}$ ).

[64] W1999 presents growth rates plotted as a function of supersaturation $(S-1)$ in (polar and mesospheric) Martian atmospheric conditions, with no surface kinetics and no radiative transfer taken into account, at different pressures and for different particle radii. Thus, we can compare our corresponding growth rates $(\mathrm{d} r / \mathrm{d} t)_{\text {CLASSIC }}$ to W1999's (Y93's) growth rates. Figures 8 and 9 display growth rates for different supersaturation $(S-1)$, in respectively mesospheric conditions $\left(p=0.02 \mathrm{~Pa}, r=100 \mathrm{~nm}, K_{n} \sim 10^{5}\right)$ and polar conditions (Figure 9) for two different radii, $(p=80$ $\left.\mathrm{Pa}, r=100 \mathrm{~nm}, K_{n} \sim 1\right)$ and ( $p=80 \mathrm{~Pa}, r=10 \mu \mathrm{m}, K_{n} \sim$ $100)$. We also show growth rates obtained with the linearized model. As an example, in the mesosphere (Figure 8) at a saturation $S=10$ for a $100 \mathrm{~nm}$ particle, the growth rate predicted by the linearized model is $4 \mu \mathrm{m} \mathrm{h}^{-1}$, while we predict
$1 \mu \mathrm{m} \mathrm{h}^{-1}$ with the CLASSIC model, and equation (25) gives $0.3 \mu \mathrm{m} \mathrm{h}^{-1}$. Interestingly, $(\mathrm{d} r / \mathrm{d} t)_{\text {CLASSIC }}$ growth rates match W1999's results for current Martian conditions. The linearized model tends to overestimate the growth rates with increasing $S$, compared to the CLASSIC model or to Y93's growth rates as derived by W1999. W1999's growth rates were reported only up to $S \sim 10$, which was identified by the author (W1999, section 8.2) as the applicability limit of Y93's equations (details in Appendix of Young [1991]).
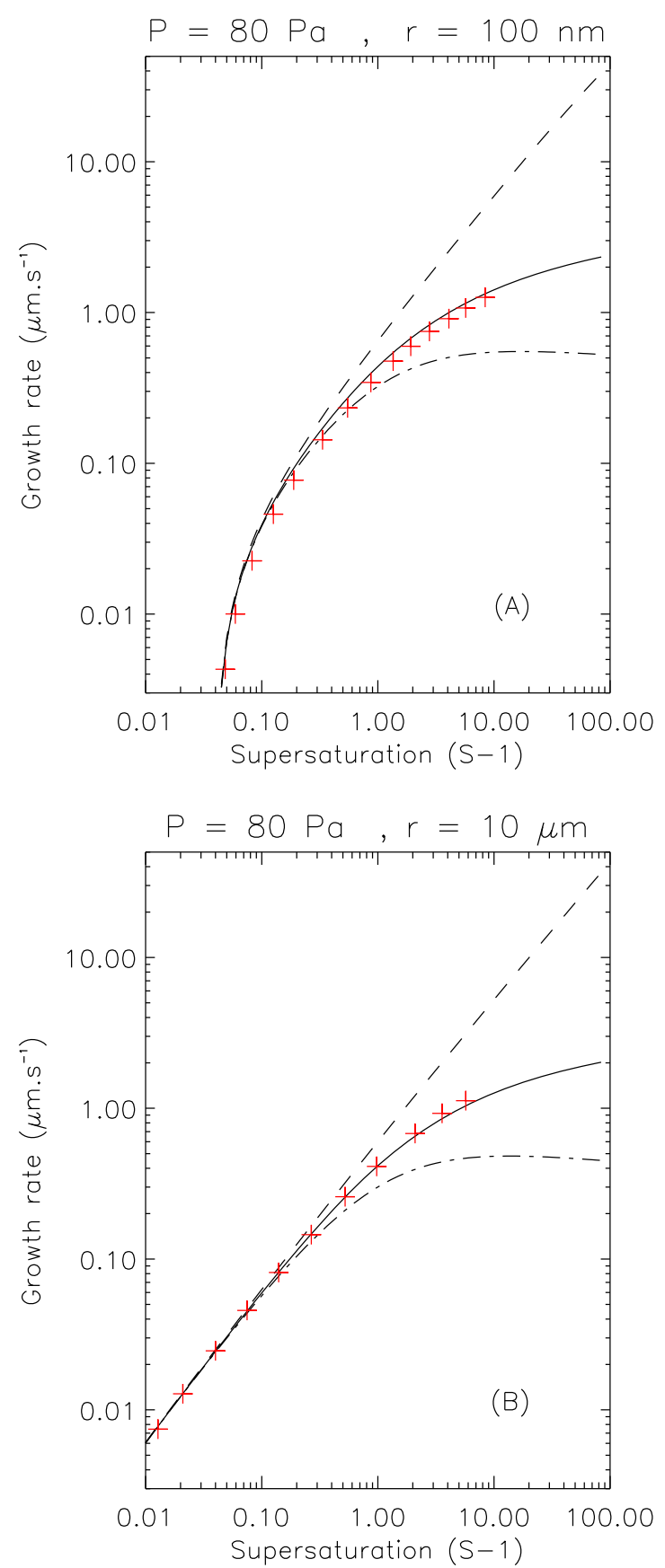

Figure 9. Same as Figure 8 with units $\mu \mathrm{m} \mathrm{s}^{-1}, p=80$ $\mathrm{Pa}$ and a particle radius (a) $r=100 \mathrm{~nm}$ and (b) $r=10$ $\mu \mathrm{m}$, at $x_{v, \infty}=95 \%$. Red crosses are extracted from W1999 (Chapter 8, Figure 8.7 for Figure 9a and Figure 8.6 for Figure 9b). 
[65] The match between the CLASSIC model and W1999 reinforces our result that Stefan flow, thermal mass diffusion, and Dufour effect are indeed negligible processes within Martian conditions. However, it is a delicate matter to strictly identify all the transport processes we investigated with the ones included in W1999. As we said the Stefan flow is indeed included in Y93's equations and we can identify the partial pressure gradient influence on the heat transfer of Y93 with the Dufour effect, as Wood [1999] identified it. However, Y93 explicitly neglected the thermal mass diffusion (see equation (9) and related text in Y93), assuming mass diffusion is dominated by the concentration induced diffusion. Regarding thermal mass diffusion, we can say that our comparison between the CLASSIC model and the SFTHDUF model (Table 1) showed that the thermal mass diffusion was negligible within Martian conditions. But we cannot affirm that the match of the CLASSIC model (where thermal mass diffusion is not included) with W1999 model is a supplementary hint that the thermal mass diffusion is negligible since it is not included in Y93 equations, and so neither in W1999 model. We have to simply remain with the idea that the temperature gradient influence on the mass transfer as derived by $\mathrm{Y} 93$ is negligible within Martian conditions, since our CLASSIC model matches Y93 model.

[66] Interestingly, we can state that the Fuchs and Sutugin [1971] correction for free molecular regime is valid in the current Martian conditions we are investigating because the CLASSIC model built on a "Maxwell-type" approach gives the same results as W1999 model which is a Langmuir-type model valid for all Knudsen numbers. The Fuchs and Sutugin correction was adapted by Fuchs and Sutugin [1971] from Sahni's [1966] work on neutron transfer and was initially intended to mixtures where $M_{v} / M_{g} \ll 1$ [see Seinfeld and Pandis, 2006, section 12; Fuchs and Sutugin, 1971, section 3.2, formula 3.27] in order to preserve isotropy of scattering during collisions of vapor molecules. However, we observe here the success of this correction for a ratio $M_{v} / M_{g} \sim 1.5$. It could have actually been anticipated thanks to Monchick and Blackmore [1988], who found that the Fuchs and Sutugin formula is a "universally valid approximation" (they go up to mass $M_{v} / M_{g} \sim 10$ ) despite its initial restrictions. Our study thus supports the robustness of the Fuchs and Sutugin correction for the free molecular regime for a value of $M_{v} / M_{g} \sim 1.5$. Monchick and Blackmore's [1988] statement on the universality of Fuchs and Sutugin correction was shown for system where the condensible vapor (whatever its molecular weight) remains diluted. So without rigorously demonstrating the extension of Monchick and Blackmore's [1988] conclusion to regimes where the condensible vapor is the major component we nevertheless show that the Fuchs and Sutugin correction still applies for a near-pure vapor, at least to the $\mathrm{CO}_{2}$ condensation in current Martian conditions.

[67] For present Martian conditions $(p \lesssim 600 \mathrm{~Pa}$ and $r \lesssim 100 \mu \mathrm{m}$ ), our reference CLASSIC model is validated for modeling the $\mathrm{CO}_{2}$ ice clouds. W1999's set of equations takes into account processes that we have shown to be negligible. Thus, if surface kinetics are not considered or if they were known to be negligible (which is still an open question for mesospheric conditions), the CLASSIC model can be preferred to derive the growth rates of $\mathrm{CO}_{2}$ ice crystals. Note that for estimated range of ancient Mars' atmospheric pressures, our CLASSIC model still works, provided that the Knudsen numbers are not too small. This is because, close to or in the continuum regime, the "Schrage effect" [Young, 1993] gets prominent for a pure vapor and it should be then considered in the mass transfer (see discussion in Appendix C).

\section{Conclusion and Perspectives}

[68] In this work we have presented a new approach to model the growth of $\mathrm{CO}_{2}$ ice crystals in the Martian atmosphere. We chose as our framework a "Maxwell-type" approach based on continuum equations (Fick's law and Fourier's law) with a Fuchs and Sutugin correction to account for the reduced efficiency of transport properties, due to the transition to free molecular regime that prevails on Mars ( $K n$ always $\gtrsim 0.1$ ). Our model stands as a compromise between two previous models by Colaprete and Toon [2003] and Wood [1999]:

[69] 1. The physics remains as simple as in the model used by Colaprete and Toon [2003] and Colaprete et al. [2003, 2008]. We have tested additional processes that could have been of importance for near-pure vapor condensation (section 2.2), namely, the Stefan flow and the coupled thermal mass diffusion and Dufour effect, and we have shown that they are negligible, whatever the saturation ratios relevant to the Martian atmosphere.

[70] 2. The mathematics requires an iterative solution as in Wood [1999] because, in contrary to Colaprete and Toon [2003], we do not rely on the linearization of the saturation vapor pressure exponential dependance. High temperature differences prevent from doing this simplification. Indeed, $\Delta T=T_{a}-T_{\infty} \gtrsim 1 \mathrm{~K}$ can occur in situation of high saturations ratios as encountered in the Martian mesosphere and prohibits using the aforementioned linearization. In addition, a near-pure vapor that is condensing is responsible for potentially higher mole fraction gradients $\left(\Delta x_{v}\right)$ than in the case of a trace gas and is thus responsible for a higher mass transfer rate $\left(I_{m}\right)$ and a higher temperature gradient $\Delta T$. Importantly, the reason for a high $\Delta T=T_{a}-T_{\infty}$ is also that the kinetically limited regime inhibits efficient removal of heat from the crystal surface.

[71] We have shown that the heritage of Earth trace gas growth rate expressions (linearized model) can lead to overestimations of growth rates in the case of a near-pure vapor condensation in a highly supersaturated environment, like $\mathrm{CO}_{2}$ in the Martian mesosphere (section 3.3). Growth rates derived with the CLASSIC model are for instance 1.2 times smaller than the linearized model at $S \sim 1.4,4$ times smaller at $S \sim 10$ and 150 times smaller at $S \sim 1000$. Note that conversely predicted evaporation rates are bigger with the CLASSIC model, compared to a linearized model. In the polar nights, regarding tropospheric $\mathrm{CO}_{2}$ ice crystals, as long as saturation ratios remain close to the mean values derived by Hu et al. [2012], ie around 1.25-1.30, overestimations of growth rates by linearized models would not exceed $\sim 10 \%$ (for $r \geq 100 \mathrm{~nm}$ ). Mesospheric $\mathrm{CO}_{2}$ ice clouds can be confronted to an extreme environment with cold pockets that can lead to values $S \sim 1000$. In this case, using a linearized model leads to a $300 \%$ overestimation of the growth rates at $S \sim 10$ for a $30 \mathrm{~nm}$ crystal for instance. The consequences of such differences remain to be tested within a full 1-D 
microphysical model (ongoing work) in order to assess the real influence of using one or the other growth rate on, e.g., the effective sizes, extension, and life time of the clouds, in case of a highly supersaturated regime.

[72] The match between our growth rate model and Young's [1993] model as used by Wood [1999] (with no surface kinetics considerations) is also due to the robustness of the Fuchs and Sutugin correction which is originally intended to vapor-gas mixture with $M_{v} / M_{g} \ll 1$, with low abundances of vapor. Here the Fuchs and Sutugin correction works well for the near-pure vapor $\mathrm{CO}_{2}$ in the Martian rarefied atmosphere, with $M_{\mathrm{CO}_{2}} / M_{\mathrm{N}_{2}} \sim 1.5$. We have thus illustrated the result of Monchick and Blackmore [1988] who showed that the Fuchs and Sutugin correction can be used for systems with the ratio $M_{v} / M_{g}$ larger than 1 .

[73] We have shown that Stefan flow, thermal mass diffusion, and Dufour effect are negligible up to pressures of $5 \times 10^{5} \mathrm{~Pa}$ so that the CLASSIC model can be used with higher atmospheric pressures relevant for ancient Mars (with some limitations related to the Knudsen number). Besides, this conclusion is valid for lower abundances as well.

[74] We propose the CLASSIC model (Table 1) for deriving the actual growth rates of $\mathrm{CO}_{2}$ ice crystals in the current Martian atmosphere as a simple but accurate approach, applicable also to the extreme saturation ratios existing in the mesosphere. Results of the CLASSIC model implementation in a 1-D microphysical model [Montmessin et al., 2002, 2004] will be shown in a future paper where we focus on the formation of $\mathrm{CO}_{2}$ ice clouds in the current Martian environment with high supersaturations, as observed in the mesosphere. Radiative heat transfer which has been neglected so far in the growth rates of the present and previous studies will be discussed as a possible factor influencing $\mathrm{CO}_{2}$ ice crystal growth.

\section{Appendix A: Derivation of the Thermal Mass Diffusion Term With Stefan Flow}

[75] We want to integrate the equation giving the mass flux density with thermal mass diffusion $\left(\mathrm{kg} \mathrm{m}^{-2} \mathrm{~s}^{-1}\right)$ :

$$
\overrightarrow{j_{v}}=-\frac{D M_{v} p}{R T\left(1-x_{v}\right)}\left(\nabla x_{v}+\frac{\alpha x_{v}\left(1-x_{v}\right)}{T} \nabla T\right)
$$

[76] For that we need a relationship between $\nabla T$ and $\nabla x_{v}$. [77] In stationary regime, assuming $\rho$ and $D$ constant, the mole fraction and temperature profile for a trace gas are

$$
\begin{gathered}
x_{v}(r)=x_{v, \infty}+\left(x_{v, a}-x_{v, \infty}\right) \frac{a}{r}, \\
T(r)=T_{\infty}+\left(T_{a}-T_{\infty}\right) \frac{a}{r} .
\end{gathered}
$$

[78] Using these profiles like Kulmala and Vesala [1991], we obtain

$$
\nabla T=\frac{T_{a}-T_{\infty}}{x_{v, a}-x_{v, \infty}} \nabla x_{v}
$$

[79] Under the same assumptions and taking into account the Stefan flow, the mole fraction profile becomes

$$
x_{v}(r)=1-\left(1-x_{v, \infty}\right)\left(\frac{1-x_{v, a}}{1-x_{v, \infty}}\right)^{\frac{a}{r}} .
$$

[80] Equation (A5) can be written in the following form:

$$
x_{v}(r)=1-\left(1-x_{v, \infty}\right) \exp \left[\frac{a}{r} \ln \left(\frac{1-x_{v, a}}{1-x_{v, \infty}}\right)\right] .
$$

[81] Differentiation of equation (A6) gives

$$
\mathrm{d} x_{v}=\frac{a}{r^{2}} \ln \left(\frac{1-x_{v, a}}{1-x_{v, \infty}}\right)\left(1-x_{v}(r)\right) \mathrm{d} r .
$$

[82] Keeping equation (A3) for $T(r)$, we obtain a new relation linking $\nabla x_{v}$ and $\nabla T$ :

$$
\nabla T=-\frac{T_{a}-T_{\infty}}{1-x_{v}(r)}\left[\ln \left(\frac{1-x_{v, a}}{1-x_{v, \infty}}\right)\right]^{-1} \nabla x_{v} .
$$

[83] Using equations (A8) and (A1), we get the new mass transfer rate accounting for Stefan flow in the thermal mass diffusion term derivation:

$$
I_{m, S F T H}=-\frac{4 \pi a p M_{v} D}{R T_{\infty}}\left(s f+t h_{S F}\right)
$$

with

$$
s f=\ln \left(\frac{1-x_{v, a}}{1-x_{v, \infty}}\right)
$$

and the new thermal mass diffusion term

$$
t h_{S F}=-\alpha \frac{T_{a}-T_{\infty}}{T_{\infty}}\left(\frac{x_{v, a}-x_{v, \infty}}{\ln \left(\frac{1-x_{v, a}}{1-x_{v, \infty}}\right)}+1\right) .
$$

\section{Appendix B: Linearized Growth Rate Models and Their Application to Martian Clouds}

[84] We present in Table B1 linearized model growth rate expressions and indicate whether they have been applied to model the growth of crystals on Mars. Some authors [Barkstrom, 1978; Ramaswamy and Detwiler, 1986; Toon et al., 1989] do not present their condensational growth model with a formalism using the so-called resistances, $R_{d}$ and $R_{h}$ [Kuroda, 1984; MacKenzie and Haynes, 1992]. However, as it is just a matter of presenting the underlying algebra it is possible for comparison purposes to express all the growth rates using this notation. All formulae are expressed with the notations we use in this work and with the radiative term, if any, set to zero. Surface kinetics effects are set to zero. Note that including them would have lead to a supplementary resistance [Kuroda, 1984]: $R_{k}=1 /\left(r \times \beta\left(\sigma_{\text {surf }}\right)\right)$, where $\sigma_{\text {surf }}$ is the surface supersaturation and $\beta\left(\sigma_{\text {surf }}\right)$ the kinetic coefficient that quantifies the efficiency of vapor molecules integration into the crystal lattice.

\section{Appendix C: Limitations of the CLASSIC Growth Model for an Ancient (Denser) Martian Atmosphere}

[85] Pursuing the comparison with W1999, we get to denser atmospheric conditions. These are less of our concern as we mostly focus on present Mars. However, $\mathrm{CO}_{2}$ ice clouds being of interest for early Mars climates, we want to estimate how far our reference CLASSIC model matches W1999. Figure C1 shows growth rates for $p=10^{4}$ 
Table B1. Growth Rates of Linearized Models and Their References, as Well as Their Application to Crystal Growth in Martian Cloud Models ${ }^{\mathrm{a}}$

\begin{tabular}{|c|c|c|}
\hline Growth Rate & Reference & Martian Clouds \\
\hline$\frac{\mathrm{d} r}{\mathrm{~d} t}=\frac{1}{r} \frac{S-1}{R_{d}+R_{h}+R_{k}(r)}$ & $\begin{array}{c}\text { Kuroda }[1984]^{\mathrm{b}} \\
\text { MacKenzie and Haynes }[1992]^{\mathrm{c}}\end{array}$ & $\begin{array}{c}\mathrm{H}_{2} \mathrm{O} \text { ice clouds with } R_{k}(r)=0 \\
{[\text { Montmessin et al. }, 2002]} \\
\mathrm{CO}_{2} \text { ice polar clouds with } R_{d}=0 \\
{[\text { Tobie et al., 2003] }]^{\mathrm{d}}}\end{array}$ \\
\hline$\frac{\mathrm{d} r}{\mathrm{~d} t}=\frac{1}{r} \frac{S-S_{e q}}{R_{d \frac{1}{\sqrt{2}}+R_{h} \frac{1}{f_{1}}}}$ & $\begin{array}{c}\text { Barkstrom }[1978]^{\mathrm{e}} \\
\text { Ramaswamy and Detwiler }[1986]^{\mathrm{f}}\end{array}$ & $\begin{array}{c}\text { no direct application } \\
\text { used by Toon et al. [1989] }\end{array}$ \\
\hline$\frac{\mathrm{d} r}{\mathrm{~d} t}=\frac{1}{r} \frac{S-S_{e q}}{R_{d} \frac{1}{f_{v}}+R_{h} \frac{1}{f_{t}} \times S}$ & Toon et al. $[1989]^{\mathrm{g}}$ & $\begin{array}{c}\mathrm{H}_{2} \mathrm{O} \text { ice clouds } \\
{[\text { Michelangeli et al., 1993] }} \\
{[\text { Colaprete et al., 1999] }} \\
{[\text { Pathak et al., 2008] }} \\
{[\text { Daerden et al., 2010] }} \\
\mathrm{CO}_{2} \text { ice clouds } \\
{[\text { Colaprete and Toon, 2003] (Early Mars) }} \\
{[\text { Colaprete et al. }, 2003] \text { (Poles) }} \\
{[\text { Colaprete et al., 2008] (Poles and Mesosphere) }}\end{array}$ \\
\hline$\frac{d r}{d t}=\frac{1}{r} \frac{S-S_{e q}}{R_{d}+R_{h} \times S_{e q}}$ & see first row & $\begin{array}{c}\mathrm{H}_{2} \mathrm{O} \text { ice clouds } \\
{[\text { Montmessin et al., 2004] }} \\
\text { Present work: section 3.3.1 only }\end{array}$ \\
\hline
\end{tabular}

aAll growth rates are reexpressed with the "resistances" notations [Kuroda, 1984]. $r$ is the radius of the growing crystal, $S$ the saturation ratio, and $S_{e q}$ the equilibrium saturation ratio accounting for curvature effect. $R_{d}, R_{h}$, and $R_{k}$ are the so-called resistances to growth, respectively, due to diffusion, heat conduction, and surface kinetics, as defined in section 3.3.1. The effective correction for free molecular regime is included in the resistances, through $D$ and $K$.

${ }^{\mathrm{b}}$ General study of ice crystals. No curvature effect taken into account.

'Study of stratospheric ice crystals. The authors introduce a radiative term, $[R A D]$, set here to 0 . No curvature effect taken into account.

"The authors alternatively consider a "fast growth" scenario limited by heat transfer (and then $R_{k}=0$ ) and "slow growth" one limited by surface kinetics (and then $R_{h}=0$ ).

${ }^{\text {e }}$ Study of cloud droplets. The author introduces a radiative term $(Q)$ set here to $0 . f_{1}$ and $f_{2}$ are defined as the corrections for the free molecular regimes, respectively, for particular diffusion and heat transfer. $S_{e q}$ is the equilibrium saturation over a crystal of radius $r$, accounting for the curvature effect.

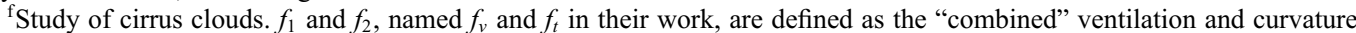
factor.

${ }^{g}$ Study of polar stratospheric ice clouds. $f_{v}$ and $f_{t}$ are defined as the ventilation and curvature factor only. $R_{h}$ is multiplied by $S$ instead of $S_{e q}$ (see section 3.3.3 for details).

Pa with, respectively, $r=100 \mathrm{~nm}$ (Figure C1a) and $r=10$ $\mu \mathrm{m}$ (Figure $\mathrm{C} 1 \mathrm{~b}$ ), this time assuming an atmosphere with $x_{v}=100 \%$ (pure vapor). The match is still very good for $r=100 \mathrm{~nm}$ and $x_{v}=99 \%$ which corresponds to $K n \sim 1$. (Taking $100 \%$ in our model would have no physical sense since it relies on diffusion processes, which cannot act in pure vapor.) For $r=10 \mu \mathrm{m}(K n \sim 0.01)$, it can be seen on Figure $\mathrm{C} 1 \mathrm{~b}$ that only the general trend is well reproduced, but a shift appears between our numerical values and Wood's values so that at a supersaturation of 4 (largest supersaturation reached by Wood for these conditions), Wood predicts $11.7 \mu \mathrm{m} \mathrm{h}^{-1}$ while we predict $8.3 \mu \mathrm{m} \mathrm{h}^{-1}$, giving a $40 \%$ difference between the models. This difference decreases with decreasing supersaturations: $33 \%, 22 \%$, and $7 \%$ at $(S-1) \sim$ $0.5,0.05$, and 0.02 , respectively. This leads us to state that our approach would lead to false results for $K n<0.1$, for supersaturations higher than $\sim 2 \%$.

[86] In the following we discuss the mismatch observed in Figure C1b between the CLASSIC model and W1999. The Stefan flow, the thermal mass diffusion, and the Dufour effect are negligible for $x_{v}=99 \%$ at $p=10^{4} \mathrm{~Pa}$ in Martian conditions (they already were for $x_{v}=95 \%$ as shown in Figure 5). Thus, the explanation for the discrepancy cannot lie in the fact that we do not include these additional processes. Seinfeld and Pandis [2006] mention different corrections of transport properties to account for the transition to kinetic regimes. Among these, except from the Fuchs and Sutugin correction, only the Dahneke approach [Dahneke, 1983] has a correct behavior near the kinetic regime and could have been suitable to our case. However, the shapes of these two correcting functions are similar for all Knudsen numbers and the Dahneke approach would not change our conclusions. Thus, the explanation has nothing to do with the correction we choose among the ones that have been derived.

[87] We provide now two plausible explanations for this difference between the CLASSIC model and W1999, at $K n<0.1$. The first reason could be that the use of the Fuchs and Sutugin correction (or any other available correction) is questionable here. It is possible that this correction does not hold anymore for a near-pure vapor at $K n<0.1$, too close to the continuous regime. The isotropy of scattering of colliding vapor molecules, as required for the Fuchs and Sutugin correction to apply, would not hold anymore for a near pure vapor in a transition regime too close to the continuum regime. A second reason could lie in the so-called "Schrage effect" [Young, 1993] which is named after the work of Schrage [1953]. Schrage [1953] was interested in interphase mass transfer in a pure vapor (e.g., from the vapor 

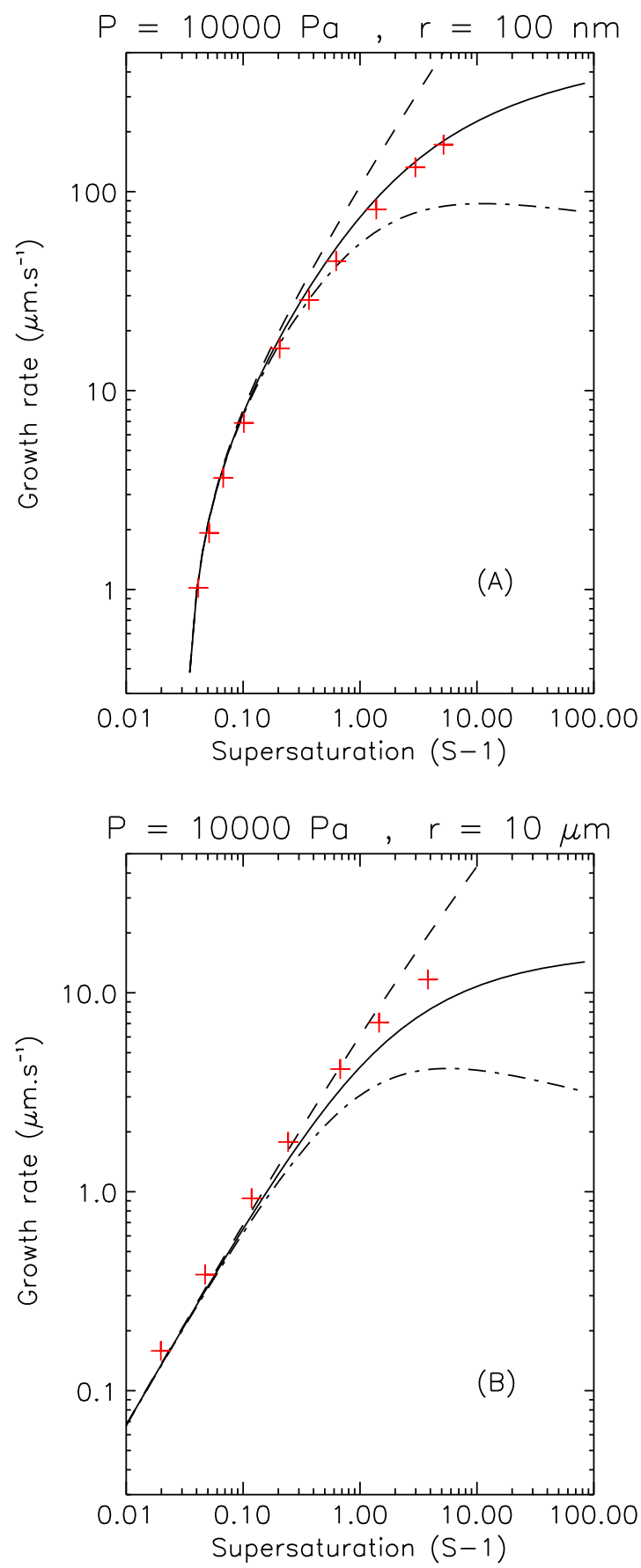

Figure C1. Same as Figure 8 with units $\mu \mathrm{m} \mathrm{s}^{-1}, p=10^{4} \mathrm{~Pa}$ and a particle radius (a) $r=100 \mathrm{~nm}$ and (b) $r=10 \mu \mathrm{m}$, at $x_{v, \infty}=99 \%$. Red crosses are extracted from W1999 (Chapter 8, Figure 8.8 for Figure C1a and Figure 8.9 for Figure $\mathrm{C} 1 \mathrm{~b})$.

phase to the ice phase), regardless of any diffusion processes (intraphase transfer). The "Schrage effect" is the enhancement of the mass flux density due to the bulk motion of the condensing pure vapor, where only interphase transfer occurs (in opposition to intraphase transfer, not considered by Schrage [1953]). This effect is included in Y93 model but not in our CLASSIC model since we only consider intraphase processes. Moreover, Y93 notes that the "Schrage effect (...) decreases in importance with increasing Knudsen number." Thus, this effect fades with increasing $K n$ and would be negligible for current Martian conditions ( $K n \geq 1)$. In the case of Figure $\mathrm{C} 1 \mathrm{~b}$, where $K n$ gets lower than 0.1 , this effect could be the cause of the disagreement between our CLASSIC model and W1999. Indeed, the latter obtains higher growth rates than our reference model. This second explanation does not preclude the first one. Both might be relevant to explain the discrepancy between the CLASSIC model and W1999, that we observe for a denser atmosphere where $K n<0.1$ for $r=10 \mu \mathrm{m}$.

[88] Acknowledgments. We thank the reviewers, Stephen E. Wood and anonymous, for careful reading and very useful comments that helped to clarify the manuscript. C.L. thanks the Scientific Committee of the "Solar System" Section (Pôle "Système solaire") of Institut Pierre Simon de Laplace (IPSL) for granting financial support.

\section{References}

Azreg-Aïnou, M. (2005), Low-temperature data for carbon dioxide, Monatshefte für Chemie, 136, 2017-2027.

Barkstrom, B. (1978), Some effects of 8-12 ym radiant energy transfer on the mass and heat budgets of cloud droplets, Am. Meteorol. Soc., 35, $665-673$.

Bird, R. B., W. E. Steward, and E. N. Lightfoot (1960), Transport Phenomena, Wiley, New York.

Chapman, S., and T. G. Cowling (1970), The Mathematical Theory of NonUniform Gases, Cambridge Univ. Press, Cambridge.

Clancy, R. T., and B. J. Sandor (1998), $\mathrm{CO}_{2}$ ice clouds in the upper atmosphere of Mars, Geophys. Res. Lett., 25(4), 489-492.

Clancy, R. T., M. J. Wolff, and P. R. Christensen (2003), Mars aerosol studies with the MGS TES emission phase function observations: Optical depths, particles sizes, and ice cloud types versus latitude and solar longitude, J. Geophys. Res., 108, 5098, doi:10.1029/2003JE002058.

Clancy, R. T., M. J. Wolff, B. A. Whitney, B. A. Cantor, and M. D. Smith (2007), Mars equatorial mesospheric clouds: Global occurrence and physical properties from Mars Global Surveyor Thermal Emission Spectrometer and Mars Orbiter Camera limb observations, J. Geophys. Res., 112, E04004, doi:10.1029/2006JE002805.

Colaprete, A., and O. B. Toon (2003), Carbon dioxide clouds in an early dense Martian atmosphere, J. Geophys. Res., 108(E4), 5025, doi:10.1029/2002JE001967.

Colaprete, A., O. B. Toon, and J. A. Magalhaes (1999), Cloud formation under Mars Pathfinder conditions, J. Geophys. Res., 104(E4), 9043-9053, doi:10.1029/1998JE900018.

Colaprete, A., R. M. Haberle, and O. B. Toon (2003), Formation of convective carbon dioxide clouds near the south pole of Mars, J. Geophys. Res., 108(E7), 5081, doi:10.1029/2003JE002053.

Colaprete, A., J. R. Barnes, R. M. Haberle, and F. Montmessin (2008), $\mathrm{CO}_{2}$ clouds, CAPE and convection on Mars: Observations and general circulation modeling, Planet. Space Sci., 56, 150-180, doi:10.1016/j.pss.2007.08.010.

Daerden, F., J. A. Whiteway, R. Davy, C. Verhoeven, L. Komguem, C. Dickinson, P. A. Taylor, and N. Larsen (2010), Simulating observed boundary layer clouds on Mars, Geophys. Res. Lett., 37, L04203, doi:10.1029/2009GL041523.

Dahneke, B. (1983), Simple kinetic theory of Brownian diffusion in vapors and aerosols, in Theory of Dispersed Multiphase Flow, vol. 2, edited by R. E. Meyer, pp. 97-133, Academic Press, New York.

Drossart, P., M. A. López-Valverde, M. Comas-Garcia, T. Fouchet, R. Melchiorri, J. P. Bibring, Y. Langevin, and B. Gondet (2006), Limb observations of infrared fluorescence of $\mathrm{CO}_{2}$ from OMEGA/Mars Express, in Mars Atmosphere Modelling and Observations, edited by F. Forget et al., p. 611, LMD, IAA, AOPP, CNES, ESA, Granada, Spain.

Elliot, J. L., et al. (1998), Global warming on Triton, Nature, 393, 765-767, doi:10.1038/31651.

Espy, P. J., and H. Jutt (2002), Equilibrium temperature of water-ice aerosols in the high-latitude summer mesosphere, J. Atmos. Sol-Terr. Phys., 64, 1823-1832, doi:10.1016/S1364-6826(02)00191-8.

Forget, F., and R. T. Pierrehumbert (1997), Warming early Mars with carbon dioxide clouds that scatter infrared radiation, Science, 278, $1273-1276$

Forget, F., F. Montmessin, J.-L. Bertaux, F. González-Galindo, S. Lebonnois, E. Quémerais, A. Reberac, E. Dimarellis, and M. A. López-Valverde (2009), The density and temperatures of the upper 
Martian atmosphere measured by stellar occultations with Mars Express SPICAM, J. Geophys. Res., 114, E01004, doi:10.1029/2008JE003086.

Forget, F., R. Wordsworth, E. Millour, J.-B. Madeleine, L. Kerber, J. Leconte, E. Marcq, and R. M. Haberle (2013), 3D modelling of the early Martian climate under a denser $\mathrm{CO}_{2}$ atmosphere: Temperatures and $\mathrm{CO}_{2}$ ice clouds, Icarus, 222, 81-99, doi:10.1016/j.icarus.2012.10.019.

Fuchs, N. A., and A. G. Sutugin (1971), Highly dispersed aerosols, in Topics in Current Aerosol Research, vol. 2, edited by G. M. Hidy and J. R. Brock, pp. 1-60, Pergamon, New York.

Fuller, E. N., P. D. Schettler, and J. C. Giddings (1966), A new method for prediction of binary gas-phase diffusion coefficients, Ind. Eng. Chem. $58,19-27$.

Fuller, E. N., K. Ensley, and J. C. Giddings (1969), Diffusion of halogenated hydrocarbons in helium. The effect of structure on collision cross sections, J. Phys. Chem., 73, 3679-3685.

Glandorf, D. L., A. Colaprete, M. A. Tolbert, and O. B. Toon (2002), $\mathrm{CO}_{2}$ snow on Mars and early Earth: Experimental constraints, Icarus, 160 , $66-72$.

González-Galindo, F., A. Määttänen, F. Forget, and A. Spiga (2011), The Martian mesosphere as revealed by $\mathrm{CO}_{2}$ cloud observations and general circulation modeling, Icarus, 216, 10-22, doi:10.1016/j. icarus.2011.08.006.

Hansen, G. B. (1997), The infrared absorption spectrum of carbon dioxide ice from 1.8 to $333 \mu \mathrm{m}, J$. Geophys. Res., 102, 21,569-21,588, doi:10.1029/97JE01875.

Hayne, P. O., D. A. Paige, J. T. Schofield, D. M. Kass, A. Kleinböhl, N. G. Heavens, and D. J. McCleese (2012), Carbon dioxide snow clouds on Mars: South polar winter observations by the Mars Climate Sounder, J. Geophys. Res., 117, E08014, doi:10.1029/2011JE004040.

Herr, K. C., and G. C. Pimentel (1970), Evidence for solid carbon dioxide in the upper atmosphere of Mars, Science, 167, 47-49, doi:10.1126/ science.167.3914.47.

Hu, R., K. Cahoy, and M. T. Zuber (2012), Mars atmospheric $\mathrm{CO}_{2}$ condensation above the north and south poles as revealed by radio occultation, climate sounder, and laser ranging observations, J. Geophys. Res., 117, E07002, doi:10.1029/2012JE004087.

Hudson, T. L., O. Aharonson, N. Schorghofer, C. B. Farmer, M. H. Hecht, and N. T. Bridges (2007), Water vapor diffusion in Mars subsurface environments, J. Geophys. Res., 112, E05016, doi:10.1029/2006JE002815.

Ivanov, A. B., and D. O. Muhleman (2001), Cloud reflection observations: Results from the Mars Orbiter Laser Altimeter, Icarus, 154, 190-206.

James, P. B., H. H. Kieffer, and D. A. Paige (1992), The seasonal cycle of carbon dioxide on Mars, in Mars, edited by H. H. Kieffer et al., pp. 934-968, Univ. of Ariz. Press, Tucson.

Kasting, J. F. (1991), $\mathrm{CO}_{2}$ condensation and the climate of early Mars, Icarus, 94, 1-13.

Kulmala, M., and T. Vesala (1991), Condensation in the continuum regime, J. Aerosol Sci., 22(3), 337-346.

Kuroda, T. (1984), Rate determining processes of growth of ice crystals from the vapour phase. Part I. Theoretical consideration, J. Meteorol. Soc. Jpn., 62, 552-561.

Langmuir, I. (1915), The dissociation of hydrogen into atoms. Part II: Calculation of the degree of dissociation and the heat of formation, J. Am. Chem. Soc., 37, 417-458.

Lellouch, E., T. Encrenaz, T. de Graauw, S. Erard, P. Morris, J. Crovisier, H. Feuchtgruber, T. Girard, and M. Burgdorf (2000), The 2.4-45 $\mu \mathrm{m}$ spectrum of Mars observed with the infrared space observatory, Planet. Space Sci., 48, 1393-1405, doi:10.1016/S0032-0633(00)00118-5.

Lemmon, E. W., and R. T. Jacobsen (2004), Viscosity and thermal conductivity equations for nitrogen, oxygen, argon, and air, Int. J. Thermophys., 25, 21-69, doi:10.1023/B:IJOT.0000022327.04529.f3.

López-Valverde, M. A., M. López-Puertas, J. J. López-Moreno, V. Formisano, D. Grassi, A. Maturilli, E. Lellouch, and P. Drossart (2005), Analysis of $\mathrm{CO}_{2}$ non-LTE emissions at $4.3 \mu \mathrm{m}$ in the Martian atmosphere as observed by PFS/Mars Express and SWS/ISO, Planet. Space Sci., 53, 1079-1087, doi:10.1016/j.pss.2005.03.007.

López-Valverde, M. A., F. González-Galindo, and M. López-Puertas (2011), Revisiting the radiative balance of the mesosphere of Mars, in The Fourth International Workshop on the Mars Atmosphere: Modelling and Observation, edited by F. Forget and E. Millour, pp. 359-362, Scientific Committee, Paris, France.

Määttänen, A., H. Vehkamäki, A. Lauri, S. Merikallio, J. Kauhanen, H. Savijärvi, and M. Kulmala (2005), Nucleation studies in the Martian atmosphere, J. Geophys. Res., 110, E02002, doi:10.1029/2004JE002308.

Määttänen, A., et al. (2010), Mapping the mesospheric $\mathrm{CO}_{2}$ clouds on Mars: MEx/OMEGA and MEx/HRSC observations and challenges for atmospheric models, Icarus, 209, 452-469, doi:10.1016/j.icarus.2010.05.017.

Määttänen, A., K. Pérot, A. Hauchecorne, F. Montmessin, and J.-L. Bertaux (2012), A Comparison of the Mesospheric Clouds on Mars and on the Earth, LPI Contributions, 1675, 8040, Boulder, Colo.
MacKenzie, A. R., and P. H. Haynes (1992), The influence of surface kinetics on the growth of stratospheric ice crystals, J. Geophys. Res., 97, 8057-8064, doi:10.1029/91JD01436.

Mathur, B. P., and S. C. Saxena (1966), A new method for calculation of diffusion coefficients of multicomponent gas mixtures, Ind. J. Pure Appl. Phys., 4(7), 266.

Maxwell, J. C. (1890), The Scientific Papers of James Clerk Maxwell, vol. 2, 636 pp., Dover Publ., New York, originally published in Encyclopedia Britannica, 82 pp., 1877.

McConnochie, T. H., J. F. Bell, D. Savransky, M. J. Wolff, A. D. Toigo, H. Wang, M. I. Richardson, and P. R. Christensen (2010), THEMISVIS observations of clouds in the Martian mesosphere: Altitudes, wind speeds, and decameter-scale morphology, Icarus, 210, 545-565, doi:10.1016/j.icarus.2010.07.021

Michelangeli, D. V., O. B. Toon, R. M. Haberle, and J. B. Pollack (1993), Numerical simulations of the formation and evolution of water ice clouds in the Martian atmosphere, Icarus, 100, 261-285.

Mischna, M. A., J. F. Kasting, A. Pavlov, and R. Freedman (2000), Influence of carbon dioxide clouds on early Martian climate, Icarus, 145, 546-554, doi:10.1006/icar.2000.6380.

Monchick, L., and R. Blackmore (1988), A variation calculation of the rate of evaporation of small droplets, J. Aerosol Sci., 19, 273-286.

Montmessin, F., P. Rannou, and M. Cabane (2002), New insights into Martian dust distribution and water-ice cloud microphysics, J. Geophys. Res., 107, 5037, doi:10.1029/2001JE001520

Montmessin, F., F. Forget, P. Rannou, M. Cabane, and R. M. Haberle (2004), Origin and role of water ice clouds in the Martian water cycle as inferred from a general circulation model, J. Geophys. Res., 109, E10004, doi:10.1029/2004JE002284.

Montmessin, F., et al. (2006), Subvisible $\mathrm{CO}_{2}$ clouds detected in the mesosphere of Mars, Icarus, 183, 403-410.

Montmessin, F., B. Gondet, J.-P. Bibring, Y. Langevin, P. Drossart, F. Forget, and T. Fouchet (2007), Hyper-spectral imaging of convective $\mathrm{CO}_{2}$ ice clouds in the equatorial mesosphere of Mars, J. Geophys. Res., 112, E11S90, doi:10.1029/2007JE002944.

Montmessin, F., F. Forget, J.-L. Bertaux, A. Spiga, and A. Määttänen (2011), Existence of supercold atmospheric layers in the Martian mesosphere, in Mars Atmosphere: Modelling and Observation, edited by F. Forget and E. Millour, pp. 404-405, http://adsabs.harvard.edu/abs/ 2011mamo.conf..404M

Neumann, G. A., D. E. Smith, and M. T. Zuber (2003), Two Mars years of clouds detected by the Mars Orbiter Laser Altimeter, J. Geophys. Res., 108, 5023, doi:10.1029/2002JE001849.

Owen, T. (1992), The composition and early history of the atmosphere of Mars, in Mars, edited by H. H. Kieffer et al., pp. 818-834, Univ. of Arizona Press, Tucson.

Pathak, J., D. V. Michelangeli, L. Komguem, J. Whiteway, and L. K. Tamppari (2008), Simulating Martian boundary layer water ice clouds and the lidar measurements for the Phoenix mission, J. Geophys. Res., 113, E00A05, doi:10.1029/2007JE002967.

Pettengill, G. H., and P. G. Ford (2000), Winter clouds over the north Martian polar cap, Geophys. Res. Lett., 27(5), 609-612.

Poling, B. E., J. M. Prausnitz, and J. P. O'Connell (2001), The Properties of Gases and Liquids, 5th ed. McGraw-Hill, New York.

Pollack, J. B. (1979), Climatic change on the terrestrial planets, Icarus, 37, 479-553, doi:10.1016/0019-1035(79)90012-5.

Pollack, J. B., J. M. Schwartz, and K. Rages (1990), Scatterers in Triton's atmosphere-Implications for the seasonal volatile cycle, Science, 250, 440-443, doi:10.1126/science. 250.4979 .440 .

Pruppacher, H. R., and J. D. Klett (2010), Microphysics of Clouds and Precipitation. Second Revised and Expanded Edition With an Introduction to Cloud Chemistry and Cloud Electricity, Springer Dordrecht Heidelberg, London, New York.

Ramaswamy, V., and A. Detwiler (1986), Interdependence of radiation and microphysics in cirrus clouds, J. Atmos. Sci.,43, 2289-2301, doi:10.1175/1520-0469(1986)043<2289:IORAMI>2.0.CO;2.

Rapp, M., and G. E. Thomas (2006), Modeling the microphysics of mesospheric ice particles: Assessment of current capabilities and basic sensitivities, J. Atmos. Sol.-Terr. Phys., 68, 715-744, doi:10.1016/ j.jastp.2005.10.015

Sahni, D. C. (1966), The effect of a black sphere on the flux distribution in an infinite moderator, J. Nucl. Energy AB, 20, 915-920.

Scalabrin, G., P. Marchi, F. Finezzo, and R. Span (2006), A reference multiparameter thermal conductivity equation for carbon dioxide with an optimized functional form, J. Phys. Chem. Ref. Data, 35, 1549-1575, doi:10.1063/1.2213631

Schofield, J. T., J. R. Barnes, D. Crisp, R. M. Haberle, S. Larsen, J. A. Magalhaes, J. R. Murphy, A. Seiff, and G. Wilson (1997), The Mars Pathfinder Atmospheric Structure Investigation/Meteorology (ASI/MET) Experiment, Science, 278, 1752-1757. 


\section{LISTOWSKI ET AL.: $\mathrm{CO}_{2}$ ICE CRYSTAL GROWTH ON MARS}

Scholten, F., H. Hoffmann, A. Määttänen, F. Montmessin, B. Gondet, and E. Hauber (2010), Concatenation of HRSC colour and OMEGA data for the determination and 3D-parameterization of high-altitude $\mathrm{CO}_{2}$ clouds in the Martian atmosphere, Planet. Space Sci., 58, 1207-1214, doi:10.1016/j.pss.2010.04.015.

Schrage, R. W. (1953), A Theoretical Study of Interphase Mass Transfer, Columbia Univ. Press, Oxford.

Seinfeld, J. H., and S. N. Pandis (2006), Atmospheric Chemistry and Physics: From Air Pollution to Climate Change-Second Edition, John Wiley, New York.

Smith, P. E., et al. (1997), Results from the Mars Pathfinder Camera, Science, 278, 1758-1765.

Span, R., and W. Wagner (1996), A new equation of state for carbon dioxide covering the fluid region from the triple-point temperature to $1100 \mathrm{k}$ at pressures up to $800 \mathrm{MPa}, J$. Phys. Chem. Ref. Data, 25, 1509-1596, doi:10.1063/1.555991.

Spiga, A., F. González-Galindo, M.-Á. López-Valverde, and F. Forget (2012), Gravity waves, cold pockets and $\mathrm{CO}_{2}$ clouds in the Martian mesosphere, Geophys. Res. Lett., 39, L02201, doi:10.1029/ 2011 GL050343.

Sprague, A. L., W. V. Boynton, K. E. Kerry, D. M. Janes, N. J. Kelly, M. K. Crombie, S. M. Melli, J. R. Murphy, R. C. Reedy, and A. E. Metzger (2007), Mars' atmospheric argon: Tracer for understanding Martian atmospheric circulation and dynamics, J. Geophys. Res., 112, E03S02, doi:10.1029/2005JE002597.
Tobie, G., F. Forget, and F. Lott (2003), Numerical simulation of the winter polar wave clouds observed by Mars Global Surveyor Mars Orbiter Laser Altimeter, Icarus, 164, 33-49.

Toon, O. B., R. P. Turco, J. Jordan, J. Goodman, and G. Ferry (1989), Physical processes in polar stratospheric ice clouds, J. Geophys. Res., 94, 11,359-11,380.

Vesovic, V., W. A. Wakeham, G. A. Olchowy, J. V. Sengers, J. T. R. Watson, and J. Millat (1990), The transport properties of carbon dioxide, J. Phys. Chem. Ref. Data, 19, 763-808, doi:10.1063/1.555875.

Vincendon, M., C. Pilorget, B. Gondet, S. Murchie, and J.-P. Bibring (2011), New near-IR observations of mesospheric $\mathrm{CO}_{2}$ and $\mathrm{H}_{2} \mathrm{O}$ clouds on Mars, J. Geophys. Res., 116, E00J02, doi:10.1029/2011JE003827.

Warren, S. G., W. J. Wiscombe, and J. F. Firestone (1990), Spectral albedo and emissivity of $\mathrm{CO} 2$ in Martian polar caps-Model results, J. Geophys. Res., 95, 14,717-14,741, doi:10.1029/JB095iB09p14717.

Wassiljeva, A. (1904), Wrmeleitung in gasgemischen, Phys. Z., 5(2), 737-742.

Wood, S. E. (1999), Nucleation and Growth of $\mathrm{CO}_{2}$ Ice Crystals in the Martian Atmosphere, Univ. of Calif., Los Angeles.

Young, J. B. (1991), The condensation and evaporation of liquid droplets at arbitrary Knudsen number, Int. J. Heat Mass Transfer, 34, 1649-1661.

Young, J. B. (1993), The condensation and evaporation of liquid droplets at arbitrary Knudsen number in the presence of an inert gas, Int. J. Heat Mass Transfer, 36, 2941-2956. 